\title{
1 Parallel processing in speech perception with 2 local and global representations of linguistic \\ context
}

Christian Brodbeck ${ }^{1,2, *}$, Shohini Bhattasali ${ }^{3,4}$, Aura A. L. Cruz Heredia ${ }^{3,5}$, Philip Resnik ${ }^{3,4}$, Jonathan Z. Simon ${ }^{2,6,7}$ \& Ellen Lau ${ }^{3}$

1) Department of Psychological Sciences, University of Connecticut, Storrs, CT, U.S.A.

2) Institute for Systems Research, University of Maryland, College Park, Maryland, U.S.A.

3) Department of Linguistics, University of Maryland, College Park, Maryland, U.S.A.

4) Institute for Advanced Computer Studies, University of Maryland, College Park, Maryland, U.S.A.

5) Department of Psychology, University of Pennsylvania, Philadelphia, Pennsylvania, U.S.A.

6) Department of Electrical and Computer Engineering, University of Maryland, College Park, Maryland, U.S.A.

7) Department of Biology, University of Maryland, College Park, Maryland, U.S.A.

*christianbrodbeck@me.com

Keywords: temporal response functions, entropy, surprisal, MEG

\section{Impact statement}

Speech processing engages multiple predictive models, using sublexical, word- and sentence contexts in parallel to anticipate upcoming phonemes.

\section{Abstract}

Speech processing is highly incremental. It is widely accepted that human listeners continuously use the linguistic context to anticipate upcoming concepts, words, and phonemes. However, previous evidence supports two seemingly contradictory models of how a predictive context is integrated with the bottom-up sensory input: Classic psycholinguistic paradigms suggest a twostage process, in which acoustic input initially leads to local, context-independent representations, which are then quickly integrated with contextual constraints. This contrasts with the view that the brain constructs a single coherent, unified interpretation of the input, which fully integrates available information across representational hierarchies, and thus uses contextual constraints to modulate even the earliest sensory representations. To distinguish these hypotheses, we tested magnetoencephalography responses to continuous narrative speech for signatures of local and unified predictive models. Results provide evidence that listeners employ both types of models in parallel. Two local context models uniquely predict some part of early neural responses, one based on sublexical phoneme sequences, and one based on the phonemes in the current word alone; at the same time, even early responses to phonemes also reflect a unified model 
39 that incorporates sentence level constraints to predict upcoming phonemes. Neural source local40 ization places the anatomical origins of the different predictive models in non-identical parts of 41 the superior temporal lobes bilaterally, with the right hemisphere showing a relative preference 42 for more local models. These results suggest that speech processing recruits both local and uni43 fied predictive models in parallel, reconciling previous disparate findings. Parallel models might 44 make the perceptual system more robust, facilitate processing of unexpected inputs, and serve a 45 function in language acquisition. 


\section{Introduction}

47 Acoustic events in continuous speech occur at a rapid pace, and listeners face pressure to process the speech signal rapidly and incrementally (Christiansen and Chater, 2016). One strategy that listeners employ to achieve this is to organize internal representations in such a way as to minimize the processing cost of future language input (Ferreira and Chantavarin, 2018). Different accounts have been proposed for how listeners do this, many centered on the notion that they actively predict future input (Gagnepain et al., 2012), for instance using internalized generative models (Halle and Stevens, 1962). Such predictive strategies manifest in a variety of measures that suggest that more predictable words are easier to process (Hale, 2003; Levy, 2008; Smith and Levy, 2013). For instance, spoken words are recognized more quickly when they are heard in a meaningful context (Marslen-Wilson and Tyler, 1975), and words that are made more likely by the context are associated with reduced neural responses, compared to less expected words (Holcomb and Neville, 1991; Connolly and Phillips, 1994; Van Petten et al., 1999; Diaz and Swaab, 2007; Broderick et al., 2018). This contextual facilitation is pervasive, and is sensitive to language statistics (Willems et al., 2016; Weissbart et al., 2020; Schmitt et al., 2020), as well as the discourse level meaning of the language input for the listeners (van Berkum et al., 2003; Nieuwland and Van Berkum, 2006).

In speech, words are often predictable because they occur in sequences that form meaningful messages. Similarly, phonemes are predictable because they occur in sequences that form words. For example, after hearing the beginning / $\mathrm{Alv} /, / \mathrm{3}^{\mathrm{x}} /$ would be a likely continuation forming river; /i/ would be more surprising, because riviera is a less frequent word, whereas / $\mho /$ would be highly surprising because there are no common English words starting with that sequence. Phonemes that are thus inconsistent with known word forms elicit an electrophysiological mismatch response (Gagnepain et al., 2012), and responses to valid phonemes are proportionately larger the more surprising the phonemes are (Ettinger et al., 2014; Gwilliams and Marantz, 2015; Gaston and Marantz, 2017). Predictive processing is not restricted to linguistic representations, as even responses to acoustic features in early auditory cortex reflect expectations based on the acoustic context (Singer et al., 2018; Forseth et al., 2020).

74 Thus, there is little doubt that the brain uses context to facilitate processing of upcoming input, at multiple levels of representation. Here we investigate a fundamental question about the underlying cognitive organization: Does the brain develop a single, unified representation of the input? In other words, one representation that is consistent across hierarchical levels, effectively propagating information from the sentence context across hierarchical levels to anticipate even low-level features of the sensory input such as phonemes? Or do cognitive subsystems differ in the extent and kind of context they use to interpret their input? This question has appeared in different forms, for example in early debates about whether sensory systems are modular (Fodor, 1985), or whether sensory input and contextual constraints are combined immediately in speech perception (Marslen-Wilson and Tyler, 1975; Tanenhaus et al., 1995). A similar distinction has also surfaced more recently between the local and global architectures of predictive coding 85 (Tabas and von Kriegstein, 2021).

86 A strong argument for a unified, globally consistent model comes from Bayesian frameworks, 87 which suggest that, for optimal interpretation of imperfect sensory signals, listeners ought to use 
the maximum amount of information available to them to compute a prior expectation for upcoming sensory input (Jurafsky, 1996; Norris and McQueen, 2008). An implication is that speech processing is truly incremental, with a unified linguistic representation that is updated at the phoneme (or an even lower) time scale (Smith and Levy, 2013). Such a unified representation is consistent with empirical evidence for top-down modulation of sensory representations, for example, suggesting that recognizing a word can bias subsequent phonetic representations (Luthra et al., 2021), that listeners weight cues like a Bayes-optimal observer during speech perception (Bejjanki et al., 2011; Feldman et al., 2009), and that they immediately interpret incoming speech with regard to communicative goals (Chambers et al., 2004; Heller et al., 2016). A recent implementation proposed for such a model is the global variant of hierarchical predictive coding, which assumes a cascade of generative models predicting sensory input from higher level expectations (Clark, 2013; Friston, 2010; Tabas and von Kriegstein, 2021). A unified model is also assumed by classical interactive models of speech processing, which rely on cross-hierarchy interactions to generate a globally consistent interpretation of the input (McClelland and Rumelhart, 1981; McClelland and Elman, 1986; Magnuson et al., 2018).

However, there is also evidence for incomplete use of context in speech perception. Results from cross-modal semantic priming suggest that, during perception of a word, initially multiple meanings are activated regardless of whether they are consistent with the sentence context or not, and contextually appropriate meanings only come to dominate at a later stage (Swinney, 1979; Zwitserlood, 1989). Similarly, listeners' eye movements suggest that they initially consider word meanings that are impossible given the syntactic context (Gaston et al., 2020). Such findings can be interpreted as evidence for a two-stage model of word recognition, in which an earlier retrieval process operates without taking into account the wider sentence context, and only a secondary process of selection determines the best fit with context (Altmann and Steedman, 1988). Similarly, at the sublexical level, experiments with non-words suggest that phoneme sequence probabilities can have effects that are decoupled from the word recognition process ( $\mathrm{Vi}$ tevitch and Luce, 1999, 1998). However, it is also possible that such effects occur only due to the unnaturalness of experimental tasks. For example, in the cross-modal priming task, listeners might come to expect a visual target which is not subject to sentence context constraints, and thus change their reliance on that context.

Finally, a third possibility is that a unified model coexists with more local models of context, and that they operate in a parallel fashion. For example, it has been suggested that the two hemispheres differ with respect to their use of context, with the left hemisphere relying heavily on top-down predictions, and the right hemisphere processing language in a more bottom-up man122 ner (Federmeier, 2007).

123 Distinguishing among these possibilities requires a task that encourages naturalistic engagement 124 with the context, and a non-intrusive measure of linguistic processing. To achieve this, we ana125 lyzed magnetoencephalography (MEG) responses to continuous narrative speech, using methods 126 that have previously shown electrophysiological brain responses related to predictive language models. Previous work, however, has tested either only for a local, or only for a unified context model, by either using only the current word up to the current phoneme as context (Brodbeck et al., 2018a; Gillis et al., 2021) or by using predictions from a complete history of phonemes and words (Donhauser and Baillet, 2020). Because these two context models include overlapping 
sets of constraints, their predictions for neural responses are correlated, and thus need to be assessed jointly. Furthermore, some architectures predict that both kinds of context model should affect brain responses separately. For example, a two-stage architecture predicts an earlier stage of lexical processing that is sensitive to lexical statistics only, and a later stage that is sensitive to the global sentence context. Here we directly test such possibilities by comparing the ability of different context models to jointly predict brain responses.

\section{Expressing the use of context through information theory}

The sensitivity of speech processing to different definitions of context is formalized through conditional probability distributions (Figure 1). Each distribution reflects an interpretation of ongoing speech input, at a given level of representation. We here use word forms and phonemes as units of representation (Figure 1-A), and all our predictors reflect information-theoretic quantities at the rate of phonemes; however, this is a matter of methodological convenience, and similar models could be formulated using a different granularity (Smith and Levy, 2013). Figure 1-B shows an architecture in which each level uses local information from that level, but information from higher levels does not affect beliefs at lower levels. In this architecture, phonemes are classified at the sublexical level based on the acoustic input and possibly a local phoneme history. The word level decodes the current word from the incoming phonemes, but without access to the multi-word context. Finally, the sentence level updates the sentence representation from the incoming word candidates, and thus selects those candidates that are consistent with the sentence context. In such a model, apparent top-down effects such as perceptual restoration of noisy input (Ganong, 1980; Leonard et al., 2016) are generated at higher level decision stages rather than at the initial perceptual representations (Norris, 1994). In contrast, Figure 1-C illustrates the hypothesis of a unified, global context model, in which priors at lower levels take advantage of information available at the higher levels. Here, the sentence context is used in decoding the current word by directly altering the prior expectation of the word candidates, and this sentence-appropriate expectation is in turn used to alter expectations for upcoming pho157 nemes. 
A
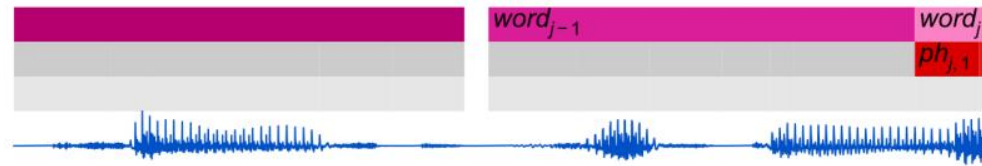

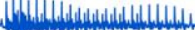

B

Local context models

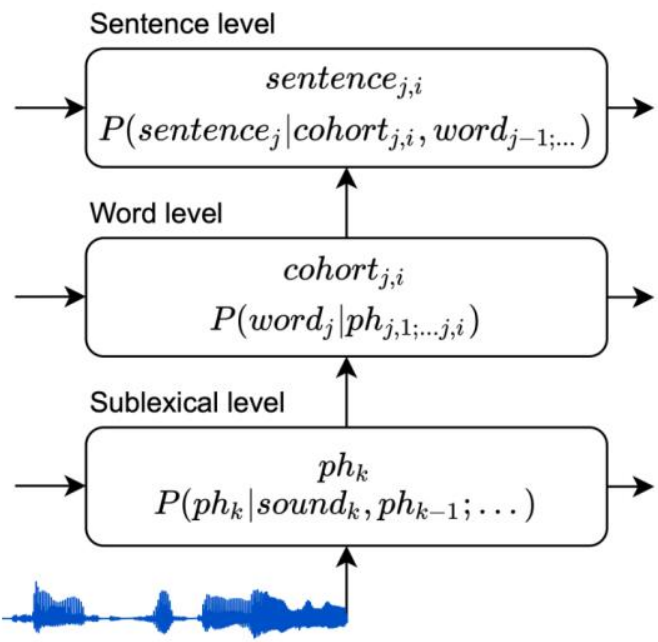

C Unified global context model

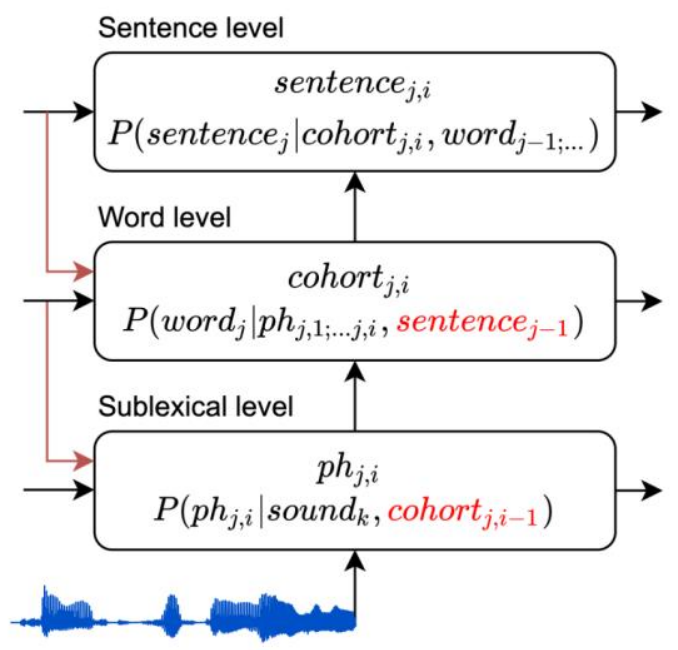

Figure 1. Information flow in local and unified architectures for speech processing.

(A) Schematic characterization of the linguistic units used to characterize speech. The same phoneme can be invoked as part of a sublexical phoneme sequence, $p h_{k}$, or as part of word $_{j}$, $p h_{j, i}$.

(B) Each box stands for a level of representation, characterized by its output and a probability distribution describing the level's use of context. For example, the sublexical level's output is an estimate of the current phoneme, $p h_{k}$, and the distribution for $p h_{k}$ is estimated as probability for different phonemes based on the sound input and a sublexical phoneme history. At the sentence level, sentence $e_{j, i}$ stands for a temporary representation of the sentence at time $j, i$. Boxes represent functional organization rather than specific brain regions. Arrows reflect the flow of information: each level of representation is updated incrementally, combining information from the same level at the previous time step (horizontal arrows) and the level below (bottom-up arrows). (C) The unified architecture implements a unified, global context model through information flowing down the hierarchy, such that expectations at lower levels incorporate information accumulated at the sentence level. Relevant differences from the local context model are in red. Note that while the arrows only cross one level at a time, the information is propagated in steps and eventually crosses all levels.

These hypotheses make different predictions for brain responses sensitive to language statistics. Probabilistic speech representations, as in Figure 1, are linked to brain activity through information theoretic complexity metrics (Hale, 2016). The most common linking variable is surprisal, which is equivalent to the difficulty incurred in updating an incremental representation of the input (Levy, 2008). Formally, the surprisal experienced at phoneme $k$ is inversely related to the likelihood of that phoneme in its context:

$$
I\left(p h_{k}\right)=-\log _{2}\left(p\left(p h_{k} \mid \text { context }\right)\right)
$$


A second information theoretic measure that has been found to independently predict brain activity is entropy (Brodbeck et al., 2018a; Donhauser and Baillet, 2020), a measure of the uncertainty in a probability distribution. Phoneme entropy is defined as the expected (average) surprisal for the next phoneme:

$$
H_{p h}\left(p h_{k}\right)=-\sum_{p h}^{\text {phonemes }} p\left(p h_{k+1}=p h \mid \text { context }\right) \log _{2}\left(p\left(p h_{k+1}=p h \mid \text { context }\right)\right)
$$

185

In contrast to surprisal, which is a function of the expected probability of the current event only, entropy is a function of the whole distribution of expectations. This makes it possible to distinguish between phoneme entropy, the uncertainty about the next phoneme, and cohort entropy, the uncertainty about the complete word form that matches the current partial input (for more details see Lexical context model in Methods):

$$
H_{w}\left(p h_{j, i}\right)=-\sum_{\text {word }}^{\text {lexicon }} p\left(\text { word }_{j}=\text { word } \mid \text { context }\right) \log _{2}\left(p\left(\operatorname{word}_{j}=\text { word } \mid \text { context }\right)\right)
$$

Entropy might relate to neuronal processes in at least two ways. First, the amount of uncertainty might reflect the amount of competition among different representations, which might play out through a neural process such as lateral inhibition (McClelland and Elman, 1986). Second, uncertainty might also be associated with increased sensitivity to bottom-up input, because the input is expected to be more informative (Jaramillo and Zador, 2011; Auksztulewicz et al., 2019).

\section{Models for responses to continuous speech}

To test how context is used in continuous speech processing, we compared the ability of three different context models to predict MEG responses, corresponding to the three levels in Figure 1-B (see Figure 2). The context models all incrementally estimate a probability distribution at each phoneme position, but they differ in the amount and kind of context they incorporate. Throughout, we used n-gram models to estimate sequential dependencies because they are powerful language models that can capture effects of language statistics in a transparent manner, with minimal assumptions about the underlying cognitive architecture (Futrell et al., 2020; Levy, 2008; Smith and Levy, 2013). An example of the complete set of predictors is shown in Figure 3.

Sublexical context model: A 5-gram model estimates the prior probability for the next phoneme given the 4 preceding phonemes. This model reflects simple phoneme sequence statistics (Vitevitch and Luce, 1999, 1998) and is unaware of word boundaries. Such a model is thought to play an important role in language acquisition (Cairns et al., 1997; Chambers et al., 2003; Saffran et al., 1996), but it is unknown whether it has a functional role in adult speech processing. The sublexical model predicted brain responses via the phoneme surprisal and entropy linking variables.

Word context model: This model implements the cohort model of word perception (MarslenWilson, 1987), applied to each word in isolation. The first phoneme of the word generates a probability distribution over the lexicon, including all words starting with the given phoneme, and each word's probability proportional to the word's relative unigram frequency. Each subsequent phoneme trims this distribution by removing words that are inconsistent with that phoneme. Like the sublexical model, the lexical model can be used as a predictive model for upcom- 
218 ing phonemes, yielding phoneme surprisal and entropy variables. In addition, the lexical model generates a probability distribution over the lexicon, which yields a cohort entropy variable.

220 Sentence context model: The sentence model is closely related to the word context model, but 221 each word's prior probability is estimated from a lexical 5-gram model. While a 5-gram model 222 misses longer-range linguistic dependencies, we use it here as a conservative approximation of 223 sentence level linguistic and interpretive constraints (Smith and Levy, 2013). The sentence model 224 implements cross-hierarchy predictions by using the sentence context in concert with the partial 225 current word to predict upcoming phonemes. Brain activity is predicted from the same three 226 variables as from the word context model.

227 We evaluated these different context models in terms of their ability to explain held-out MEG 228 responses, and the latency of the brain responses associated with each model. An architecture based on local context models, as in Figure 1-B, predicts a temporal sequence of responses as 230 information passes up the hierarchy, with earlier responses reflecting lower order context mod231 els. In contrast, a unified architecture, as in Figure 1-C, predicts that the sentence context model 232 should exhaustively explain brain responses, because all representational levels use priors de233 rived from the sentence context. Finally, architectures that entail multiple kinds of models pre234 dict that different context models might explain response components, possibly in different ana235 tomical areas.

236 Acoustic controls: In order to dissociate effects of linguistic processing from responses to acous237 tic properties of the speech stimulus, all models controlled a gammatone spectrogram and an 238 acoustic onset spectrogram (Brodbeck et al., 2020), as well as word onsets and phoneme onsets 239 (Brodbeck et al., 2018a). 


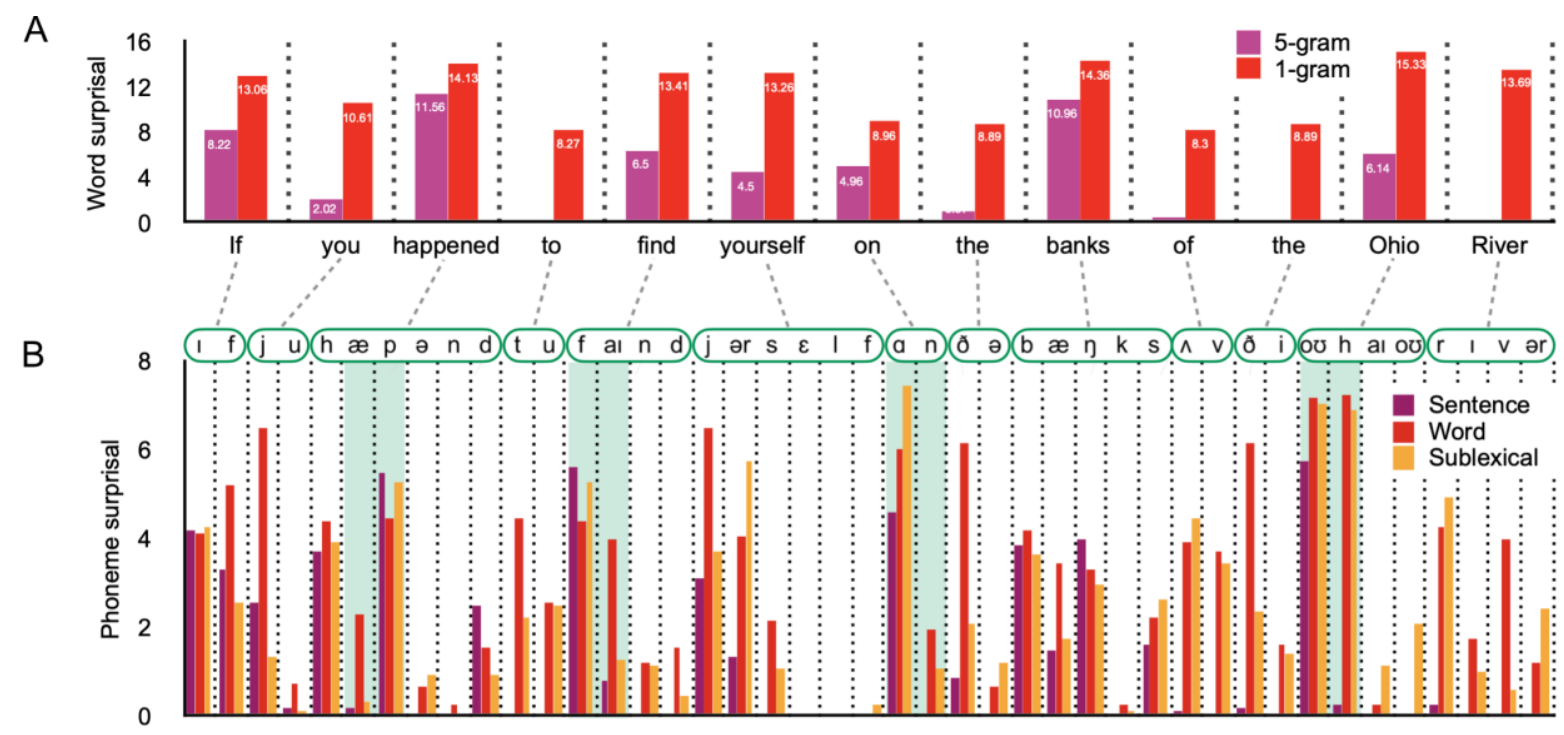

C

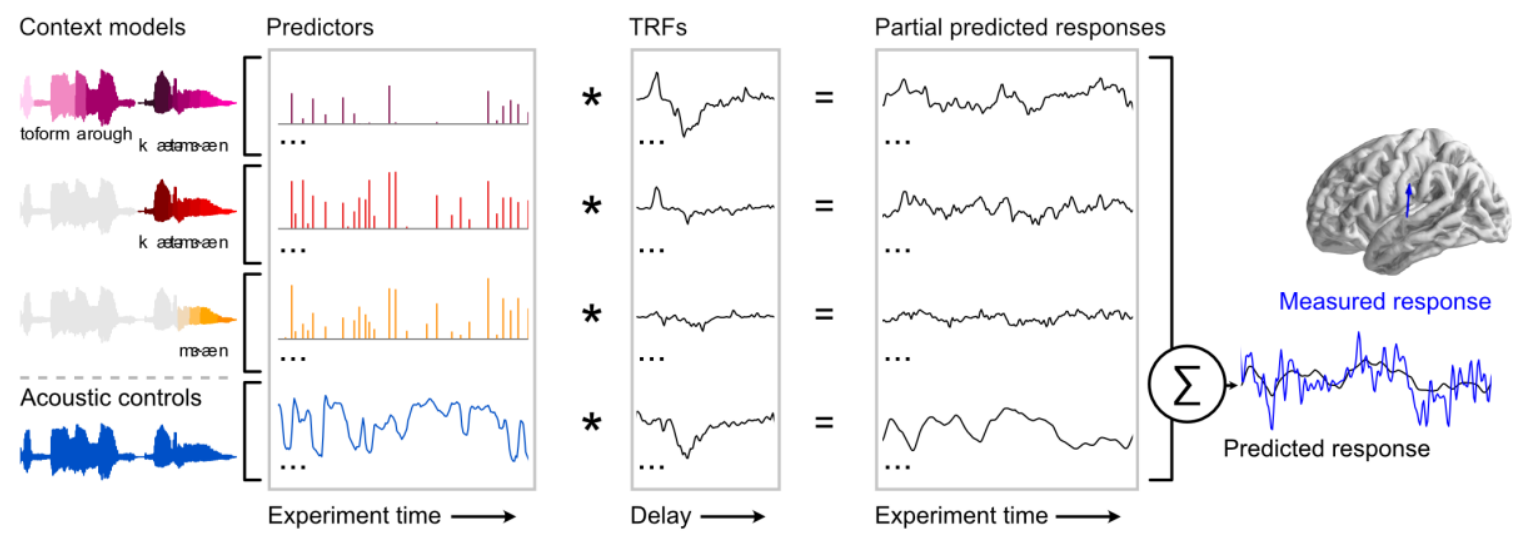

Figure 2. Models for predictive speech processing based on the sentence, word, and sublexical 242 context, used to predict MEG data.

243 (A) Example of word-by-word surprisal. The sentence (5-gram) context generally leads to a re244 duction of word surprisal, but the magnitude of the reduction differs substantially between 245 words (across all stimuli, mean \pm standard deviation, unigram surprisal: $10.76 \pm$ 5.15; 5-gram 246 surprisal: $7.43 \pm 5.98 ; t_{8172}=76.63, p<.001$ ).

247 (B) Sentence level predictions propagate to phoneme surprisal, but not in a linear fashion. For 248 example, in the word happened, the phoneme surprisal based on all three models is relatively 249 low for the second phoneme /æ/ due to the high likelihood of word candidates like have and 250 had. However, the next phoneme is /p/ and phoneme surprisal is high across all three models. 251 On the other hand, for words like find, on and Ohio, the sentence-constrained phoneme surprisal 252 is disproportionately low for subsequent phonemes, reflecting successful combination of the 253 sentence constraint with the first phoneme.

254 (C) Phoneme-by-phoneme estimates of information processing demands, based on different 255 context models, were used to predict MEG responses through multivariate temporal response 256 functions (mTRFs) (Brodbeck et al., 2018b). An mTRF consists of multiple TRFs estimated jointly 257 such that each predictor, convolved with the corresponding TRF, predicts a partial response, and 258 the point-wise sum of partial responses constitutes the predicted MEG response. The dependent 
measure (measured response) was the fixed orientation, distributed minimum norm source current estimate of the continuous MEG response. The blue arrow illustrates a single virtual current source dipole. Estimated signals at current dipoles across the brain were analyzed using a massunivariate approach. See Methods for details. TRFs: temporal response functions.

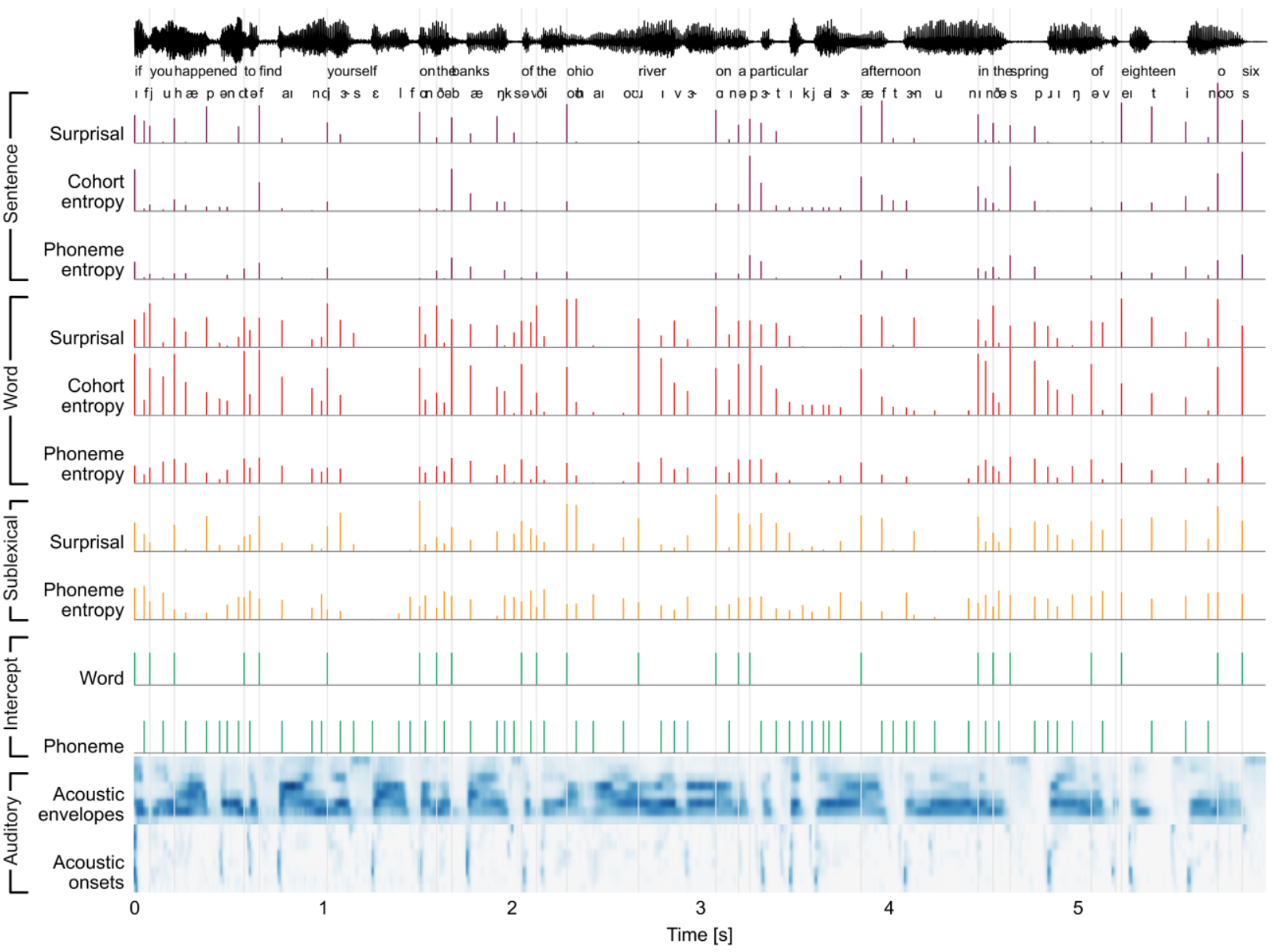

Figure 3. Stimulus excerpt with all 26 predictors used to model brain responses. From the top: Phoneme-level information-theoretic variables, based on different context definitions: Sentence, word, and sublexical context; Intercepts for word- and phoneme-related brain activity, i.e., a predictor to control for brain activity that does not scale with the variables under consideration; and an auditory processing model, consisting of an acoustic spectrogram (sound envelopes) and an onset spectrogram (sound onsets), each represented by 8 predictors for 8 frequency bands.

\section{Results}

Twelve participants listened to $\sim 47$ minutes of a nonfiction audiobook. Multivariate temporal response functions (mTRFs) were used to jointly predict held-out, source localized MEG responses (Figure 2-C). To test whether each context model is represented neurally, the predictive power of the full model including all predictors was compared with the predictive power of a model that was estimated without the predictor variables belonging to this specific context model. Besides 11 right-handers, our sample included a single left-hander. While this participant's brain responses were more right-lateralized than average, excluding them did not change the conclu- 
278 sions from any of the reported lateralization significance tests. We thus report results from the 279 total sample, but identify the left-hander in plots and source data.

280 Phoneme-, Word- and Sentence-constrained models co-exist in the brain

281 Each context model significantly improves the prediction of held-out data, even after controlling 282 for acoustic features and the other two context models (Figure 4-A). Each of the three context 283 models' source localization is consistent with sources in the superior temporal gyrus (STG), 284 thought to support phonetic and phonological processing (Mesgarani et al., 2014). In addition, 285 the sentence constrained model also extends to more ventral parts of the temporal lobe, con286 sistent with higher-level language processing (Hickok and Poeppel, 2007; Wilson et al., 2018). In 287 comparison, the predictive power of the acoustic features is highest in closer vicinity of Heschl's 288 Gyrus (Figure 4-D). At each level of context, surprisal and entropy contribute about equally to 289 the model's predictive power (Figure 4-B, Table 1).

A
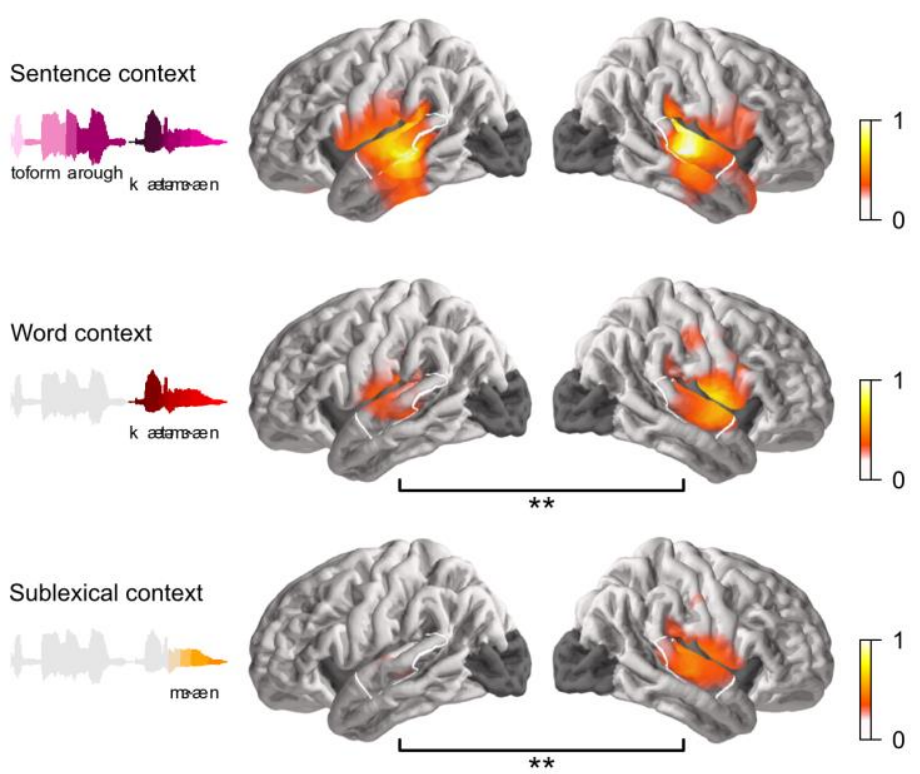

D
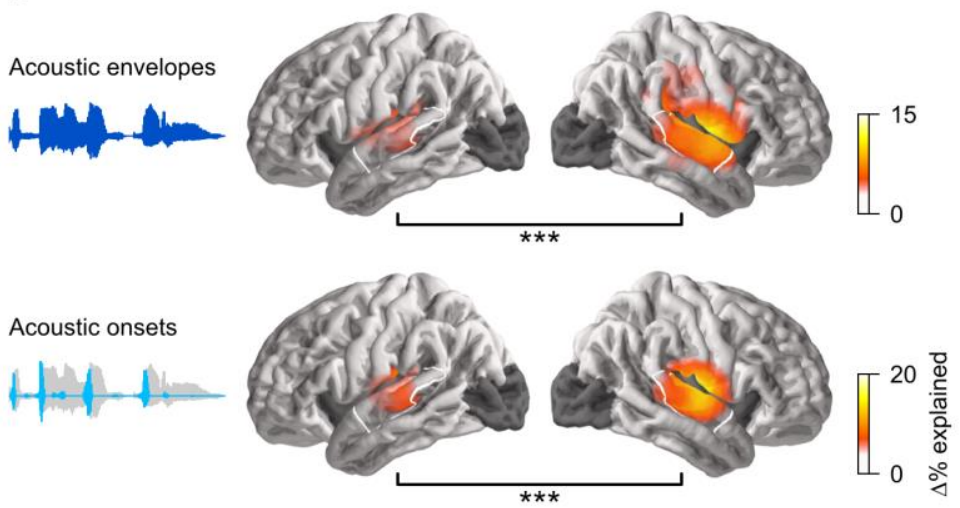

B
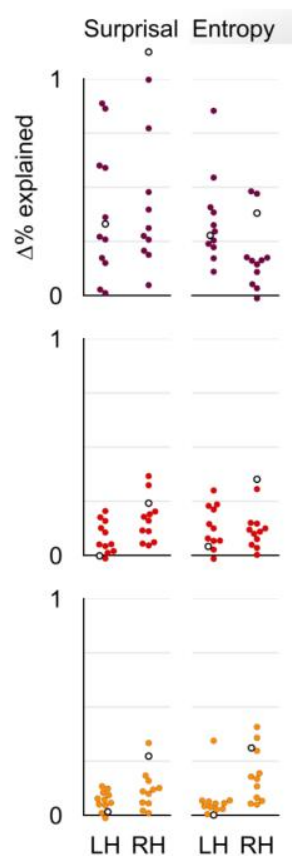

$\mathrm{E}$

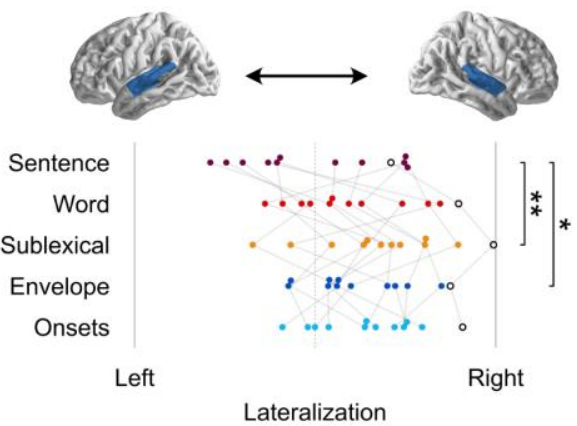

Figure 4. All context models significantly contribute to predictions of brain responses. 
(A) Each context model significantly improves predictions of held-out MEG data in both hemispheres ( $\left.t_{\max } \geq 6.16, p \leq .005\right)$. Black bars below anatomical plots indicate a significant difference between hemispheres. The white outline indicates a region of interest (ROI) used for measures shown in (B), (C) and (E). Brain regions excluded from analysis are darkened (occipital lobe and insula).

(B) Surprisal and entropy have similar predictive power in each context model. Each dot represents the difference in predictive power between the full and a reduced model for one subject, averaged in the ROI. Cohort- and phoneme entropy are combined here because the predictors are highly correlated and hence share a large portion of their explanatory power. Corresponding statistics and effect-size are given in Table 1. A single left-handed participant is highlighted throughout with an unfilled circle. LH: left hemisphere; $\mathrm{RH}$ : right hemisphere.

(C) Even when tested individually, excluding variability that is shared between the two, cohortand phoneme entropy at each level significantly improve predictions. A significant effect of sentence-constrained phoneme entropy is evidence for cross-hierarchy integration, as it suggests that sentence level information is used to predict upcoming phonemes.

307 (D) Predictive power of the acoustic feature representations.

308 (E) The lateralization index $(L I=R /(L+R))$ indicates that the sublexical context model is more 309 right-lateralized than the sentence context model. Left: $L I=0$; Right: $L I=1$.

310 Significance levels: ${ }^{*} p \leq .05 ; * * p \leq .01 ; * * * p \leq .001$. Statistics for A, D: Figure 4-Source Data 311 1.txt; ROI data for B, C, E: Figure 4-Source Data 2.txt.

Left hemisphere
\begin{tabular}{llll}
$\Delta \%$ & $t(11)$ & $\mathrm{p}$ & $\mathrm{d}$ \\
\hline
\end{tabular}
Right hemisphere

\section{Sentence context}

$\begin{array}{lllrlllll}\text { Surprisal } & 3.77 & 4.40^{* *} & .001 & 1.27 & 5.51 & 4.14^{* *} & .002 & 1.19 \\ \text { Entropy } & 3.40 & 5.96^{* * *} & <.001 & 1.72 & 1.94 & 4.11^{* *} & .002 & 1.19 \\ \text { Cohort } & 0.83 & 3.41^{* *} & .006 & 0.98 & 0.39 & 2.45^{*} & .032 & 0.71 \\ \text { Phoneme } & 0.85 & 5.18^{* * *} & <.001 & 1.50 & 0.79 & 3.85^{* *} & .003 & 1.11\end{array}$

\section{Word context}

$\begin{array}{lllllllll}\text { Surprisal } & 0.78 & 3.62^{* *} & .004 & 1.04 & 1.71 & 5.76 * * * & <.001 & 1.66 \\ \text { Entropy } & 1.26 & 4.43^{* *} & .001 & 1.28 & 1.31 & 4.39 * * & .001 & 1.27 \\ \text { Cohort } & 0.25 & 3.29 * * & .007 & 0.95 & 0.36 & 3.99 * * & .002 & 1.15 \\ \text { Phoneme } & 0.51 & 4.59 * * * & <.001 & 1.32 & 0.66 & 3.61 * * & .004 & 1.04\end{array}$

Sublexical context

313 Table 1. Predictive power in mid/posterior STG ROI for individual predictors. One-tailed t-tests and 314 Cohen's $d$ for the predictive power uniquely attributable to the respective predictors. Data un- 
317 Overall, the acoustic features explain more of the variability in brain responses than the linguistic 318 features (compare scales in Figure 4-A and D). This is likely because speech is an acoustically rich stimulus, driving many kinds of auditory receptive fields. In contrast, the linguistic predictors represent very specific computations, likely represented in a small and specialized neural territory. For the present purpose, what is critical is the consistency of the linguistic effects across subjects: Figure 4-B and $C$, as well as the effect sized shown in Table 1 suggest that the linguistic modulation of brain responses can be detected very reliably across subjects.

The significant predictive power of the local context models is inconsistent with the hypothesis of a single, unified context model (Figure 1-C). Instead, it suggests that different neural representations incorporate different kinds of context. We next pursued the question of how these different representations are organized hierarchically. Phoneme surprisal depends on the conditional probability of the current phoneme, and thus does not distinguish between whether what is predicted is a single phoneme or the whole lexical completion (Levy, 2008; Smith and Levy, 2013). Entropy, on the other hand, depends on the units over which probabilities are calculated, and can thus potentially distinguish between whether brain responses reflect uncertainty over the next phoneme alone, or uncertainty over the word currently being perceived, i.e., over the lexical completion (see Lexical context model in Methods). This distinction is particularly interesting for the sentence context model: if predictions are constrained to using context within a hierarchical level, as in Figure 1-B, then the sentence context should affect uncertainty about the upcoming word, but not uncertainty about the upcoming phoneme. On the other hand, a brain response related to sentence-conditional phoneme entropy would constitute evidence for crosshierarchy predictions, with sentence level context informing predictions of upcoming phonemes.

Even though phoneme and cohort entropy were highly correlated (sentence context: $r=.92$; word context: $r=.90$ ), each of the four representations was able to explain unique variability in the MEG responses that could not be attributed to any of the other representations (Figure 4-C, Table 1). This suggests that the sentence context model is not restricted to predicting upcoming words, but also generates expectations for upcoming phonemes. This is thus evidence for crosshierarchy top-down information flow, indicative of a unified language model that aligns representations across hierarchical levels. Together, these results thus indicate that the brain does maintain a unified context model, but that it also maintains more local context models.

All three context models individually contribute to neural representations, but are these representations functionally separable? While all three context models improve predictions in both hemispheres, the sentence constrained model does so symmetrically, whereas the lexical and sublexical models are both more strongly represented in the right hemisphere than in the left hemisphere (Figure 4-A). This pattern might suggest an overall right-lateralization of linguistic processing; however, the predictive power of the joint linguistic model (predictors from all three levels combined) is not significantly lateralized ( $\left.t_{\max }=4.11, p=.134\right)$. These results thus suggests that linguistic processing is bilateral, but that the hemispheres differ in what context models they rely on. Consistent with this, the context models differ in their relative lateralization (Figure 4-E). 
The sublexical context model is significantly more right-lateralized than the sentence model $\left(t_{11}=\right.$ $3584.41, p=.001$ ), while the word model is only numerically more right-lateralized than the sentence model $\left(t_{11}=1.53, p=.154\right)$. These lateralization patterns suggests an anatomical differentiation in the representations of different context models, with the left hemisphere primarily relying on a unified model of the sentence context, and the right hemisphere more broadly keeping track of different context levels.

Given that all three context models are represented in the STG, especially in the right hemisphere, a separate question concerns whether, within a hemisphere, the different context models predict activity in the same or different neural sources. While MEG source localization does not allow precisely separating different sources in close proximity, it does allow statistically testing whether two effects originate from the same or from a different configuration of neural sources (Lütkenhöner, 2003). The null hypothesis of such a test (McCarthy and Wood, 1985) is that a single neural process, corresponding to a fixed configuration of current sources, generates activity that is correlated with all three context models. The alternative hypothesis suggests some differentiation between the configuration of sources recruited by the different models. Results indicate that, in the right hemisphere, all three context models, as well as the two acoustic models, originate from different source configurations $\left(F_{(175,1925)} \geq 1.25, p \leq .017\right)$. In the left hemisphere, the sentence constrained model is localized differently from all other models ( $F_{(179,}$ 1969) $\geq 1.38, p<.001$ ), whereas there is no significant distinction among the other models (possibly due to lower power due to the weaker effects in the left hemisphere for all but the sentence model). In sum, these results suggest that the different context models are maintained by at least partially separable neural processes.

\section{Sentence context affects early responses and dominates late responses}

The TRFs estimated for the full model quantify the influence of each predictor variable on brain responses over a range of latencies (Figure 2-C). Figure 5 shows the response magnitude to each predictor variable as a function of time, relative to phoneme onset. For an even comparison between predictors, TRFs were summed in the anatomical region in which any context model significantly improved predictions. Note that responses prior to $0 \mathrm{~ms}$ are plausible due to coarticulation, by which information about a phoneme's identity can already be present in the acoustic signal prior to the conventional phoneme onset (Beddor et al., 2013; Salverda et al., 2003). Figure 5 Error! Reference source not found.-G shows the anatomical distribution of responses related to the different levels of context.

Surprisal quantifies the incremental update to a context model due to new input. A brain response related to surprisal therefore indicates that the sensory input is brought to bear on a neural representation that uses the corresponding context model. Consequently, the latencies of brain responses related to surprisal at different context models are indicative of the underlying processing architecture. In an architecture in which information is sequentially passed to higher level representations with broadening context models (Figure 1-B), responses should form a temporal sequence from narrower to broader contexts. However, in contrast to this prediction, the observed responses to surprisal suggest that bottom-up information reaches representations that use the sentence- and word-level contexts simultaneously, at an early response peak (Figure 5-A; peak in the early time window for sentence context: $78 \mathrm{~ms}, \mathrm{SD}=24 \mathrm{~ms}$; word context: 76 
$\mathrm{ms}, \mathrm{SD}=11 \mathrm{~ms})$. Sublexical surprisal is associated with a lower response magnitude overall, but also exhibits an early peak at $94 \mathrm{~ms}(\mathrm{SD}=26 \mathrm{~ms})$. None of these three peak latencies differ signif401 icantly (all pairwise $t_{11} \leq 2.01, p \geq .065$ ). This suggests a parallel processing architecture in which 402 different context representations are activated simultaneously by new input. Later in the 403 timecourse the responses dissociate more strongly, with a large, extended response reflecting 404 the sentence context, but not the word context starting at around $205 \mathrm{~ms}\left(t_{\max }=5.27, p=.007\right)$. 405 The lateralization of the TRFs is consistent with the trend observed for predictive power: a sym406 metric response reflecting the unified sentence context, and more right-lateralized responses 407 reflecting the more local contexts (Figure 5-B).

A Surprisal-TRF magnitude

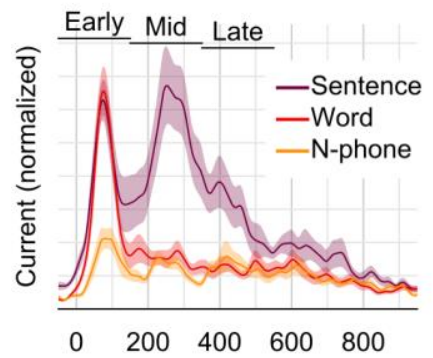

C Lexical cohort entropy

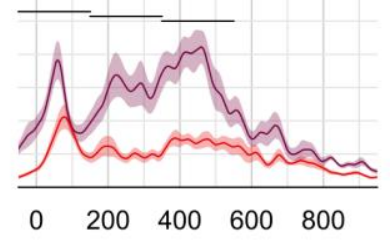

E Phoneme entropy

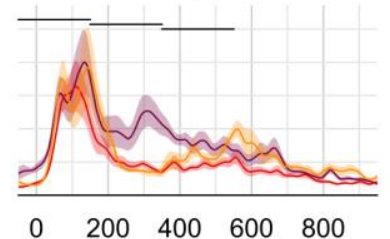

B TRF magnitude by hemisphere

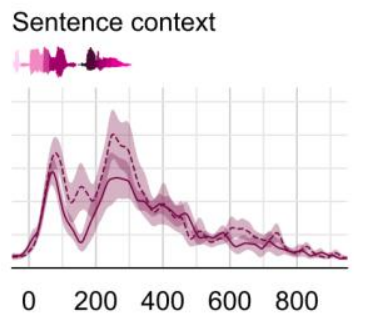

D

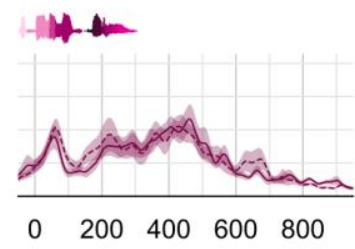

F

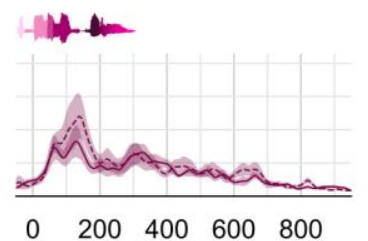

- Left hemisphere ----Right hemisphere
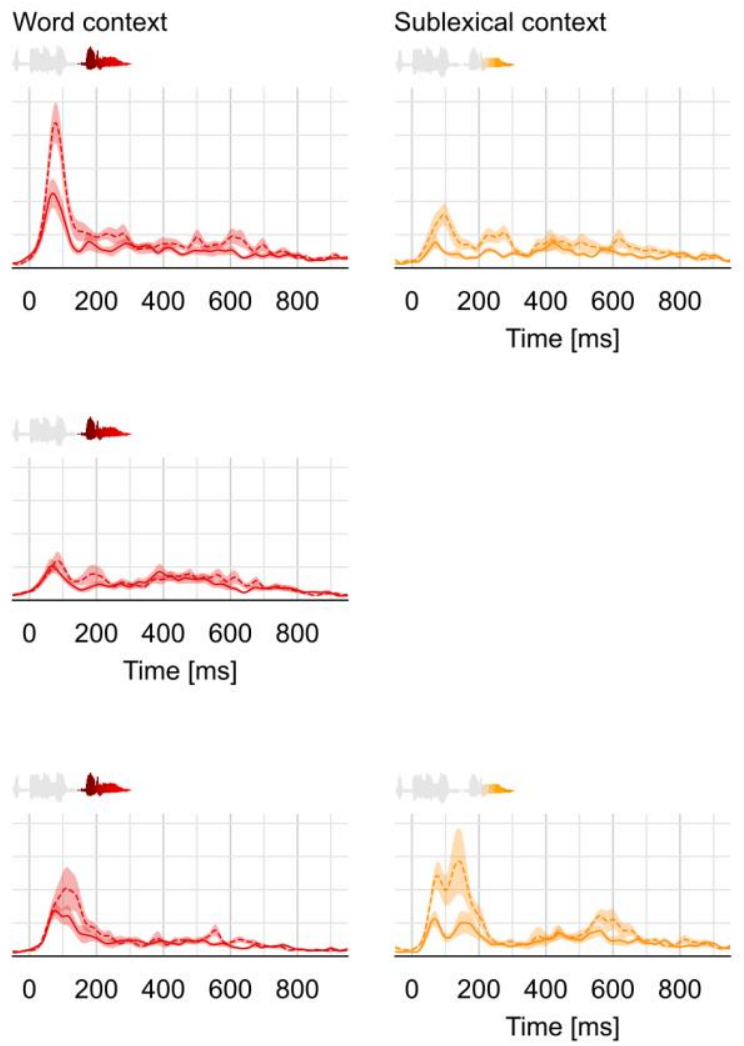

G
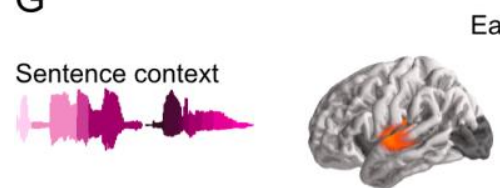

Early

Mid

Late
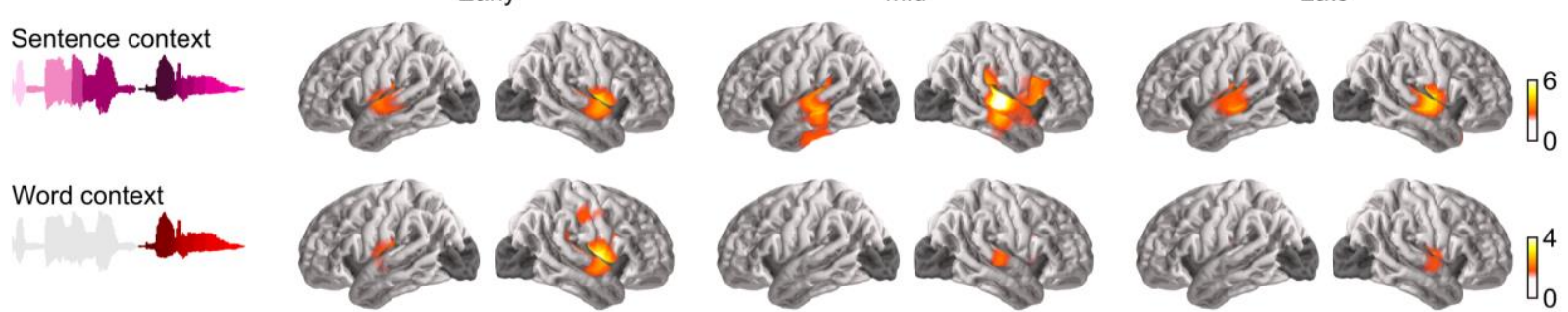

Sublexical context
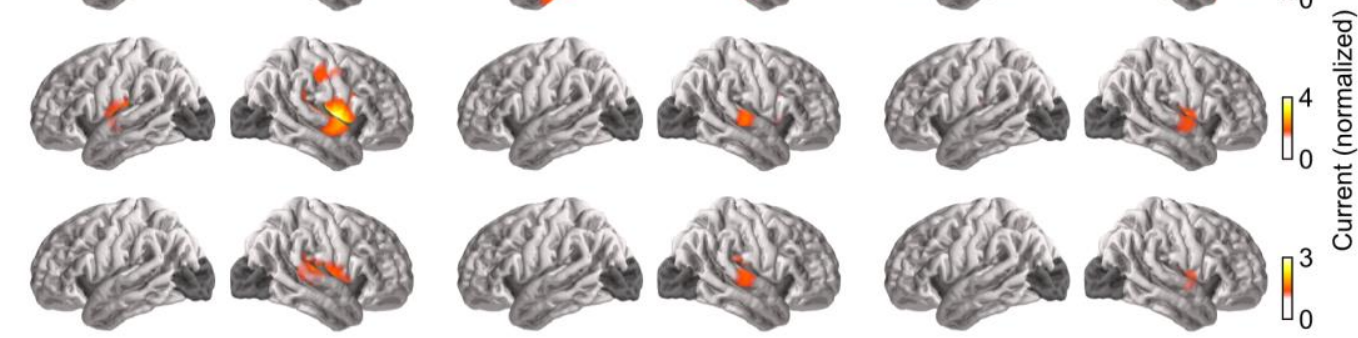
Figure 5. Early responses reflect parallel activation of all context models, later responses selective410 ly reflect activity in the sentence-constrained model.

411 (A) Current magnitude of TRFs to phoneme surprisal for each level of context (mean and within412 subject standard error (Loftus and Masson, 1994); y-axis scale identical in all panels of the fig413 ure). To allow fair comparison, all TRFs shown are from the same symmetric ROI, including all 414 current dipoles for which at least one of the three context models significantly improved the re415 sponse predictions. Bars indicate time windows corresponding to source localizations shown in 416 panel G.

417 (B) When plotted separately for each hemisphere, relative lateralization of the TRFs is consistent 418 with the lateralization of predictive power (Figure 4).

419 (C-D) TRFs to lexical cohort entropy are dominated by the sentence context model.

420 (E-F) TRFs to phoneme entropy are similar between context models, consistent with parallel use 421 of different contexts in predictive models for upcoming speech.

422 (G) All context models engage the superior temporal gyrus at early responses, mid-latency re423 sponses incorporating the sentence context also engage more ventral temporal areas. Anatomi424 cal plots reflect total current magnitude associated with different levels of context representing 425 early $(-50-150 \mathrm{~ms})$, mid-latency $(150-350 \mathrm{~ms})$ and late $(350-550 \mathrm{~ms})$ responses. The color426 scale is adjusted for different predictors to avoid images dominated by the spatial dispersion 427 characteristic of MEG source estimates.

428 Peak latencies in the early time window: Figure 5-Source Data 1.txt; Pairwise tests of TRF time 429 courses: Figure 5-Source Data 2.txt.

430 The TRFs, convolved with the corresponding predictors generate partial predicted responses (cf. 431 Figure 2-C). This reconstruction thus allows decomposing brain responses into component re432 sponses corresponding to different predictors. Figure 6 uses this to simulate the responses cor433 responding to the different context models, illustrating several of the observations made above. 434 As the sentence level was the most predictive power, so it also corresponds to higher amplitude 435 responses than the other levels. Furthermore, the sub-sentence levels exhibit small modulations 436 close to surprising phonemes, corresponding to the mainly brief, low latency TRFs. In contrast, 437 response corresponding to the sentence level are dominated by larger waves, lagging surprising 438 phonemes by several hundred milliseconds, corresponding to the sustained higher TRF ampli439 tudes at later latencies.

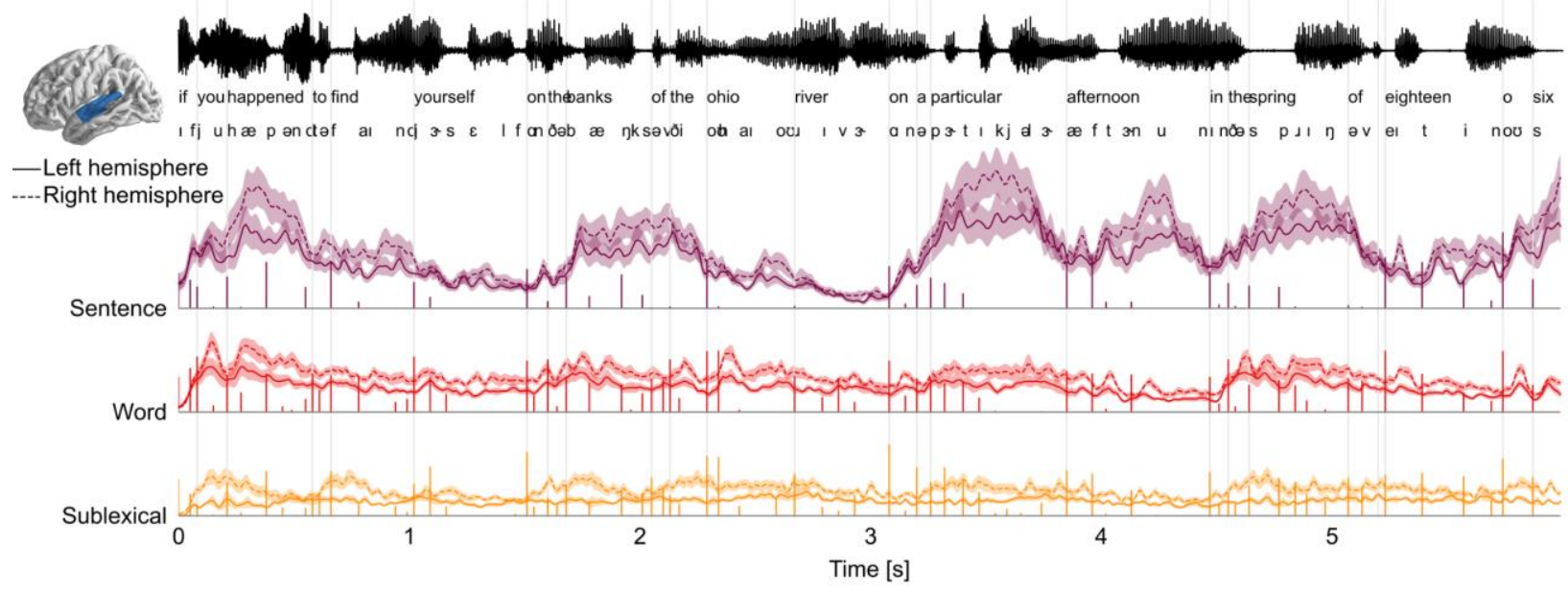


Figure 6. Decomposition of brain responses into context levels. Predicted brain responses related to processing different levels of context (combining surprisal and entropy predictors; mean and within-subject standard error). Stem plots correspond to surprisal for the given level. Slow fluctuations in brain responses are dominated by the sentence level, with responses occurring several hundred milliseconds after surprising phonemes, consistent with the high amplitude at late latencies in TRFs. Partial predicted responses were generated for each context model by convolving the TRFs with the corresponding predictors; summing the predicted responses for the predictors corresponding to the same level; and extracting the magnitude (sum of absolute values) in the STG ROI.

Sentence context dominates word recognition, all contexts drive phoneme predictions Brain responses related to entropy indicate that neural processes are sensitive to uncertainty or competition in the interpretation of the speech input. Like surprisal, such a response suggests that the information has reached a representation that has incorporated the corresponding context. In addition, because entropy measures uncertainty regarding a categorization decision, the response to entropy can distinguish between different levels of categorization: uncertainty about the current word (cohort entropy) versus uncertainty about the next phoneme (phoneme entropy).

The TRFs to cohort entropy suggest a similar pattern as those to surprisal (Figure 5 C-D). Both cohort representations are associated with an early peak (sentence context: 56 ms, SD = 28 ms; word context: $80 \mathrm{~ms}, \mathrm{SD}=23 \mathrm{~ms}$ ), followed only in the sentence constrained cohort by a later sustained effect. In contrast to surprisal, however, even early responses to cohort-entropy are dominated by the sentence context $\left(t_{\max }=5.35, p=.004\right.$ at $43 \mathrm{~ms}$; later responses: $t_{\max }=7.85, p$ $<.001$ at $461 \mathrm{~ms}$ ). This suggests that lexical representations are overall most strongly activated in a model that incorporates the sentence context.

In contrast to surprisal and cohort entropy, the responses to phoneme entropy are similar for all levels of context, dominated by an early and somewhat broader peak (Figure $5 \mathrm{E}-\mathrm{F}$ ). There is still some indication of a second, later peak in the response to sentence-constrained phoneme entropy, but this might be due to the high correlation between cohort and phoneme entropy. A direct comparison of sentence-constrained cohort and phoneme entropy indicates that early processing is biased towards phoneme entropy (though not significantly) while later processing is biased towards cohort entropy $\left(t_{\max }=4.74, p=.017\right.$ at $\left.231 \mathrm{~ms}\right)$. In sum, the entropy results suggest that all context representations drive a predictive model for upcoming phonemes. This is reflected in a short-lived response in STG, consistent with the fast rate of phonetic information. Simultaneously, the incoming information is used to constrain the cohort of word candidates matching the current input, with lexical activations primarily driven by a unified model that incorporates the sentence context. Source localization suggests that early activity originates from the vicinity of the auditory cortex in the upper STG, regardless of context (Figure 5-GError! Reference source not found.). The precise source configuration in the right STG nevertheless differs between contexts in the early time window (sentence vs word: $F_{(175,1925)}=2.08, p<.001$; word vs sublexical: $F_{(175,1925)}=5.99, p<$ 
.001). More notably, the sentence-based responses in the mid-latency window recruits more sources, localized to the middle and inferior temporal lobe. Accordingly, the sentence-based responses in the mid-latency window differs significantly from the early window (left hemisphere: $F_{(179,1969)}=1.72, p<.001$; right hemisphere: $\left.F_{(175,1925)}=5.48, p<.001\right)$. These results suggest that phonetic information initially engages a set of sources in the STG, while a secondary stage then engages more ventral sources that specifically represent the sentence context.

No evidence for a trade-off between contexts

We interpret our results as evidence that different context models are maintained in parallel. An alternative possibility is that there is some trade-off between contexts used, and it only appears in the averaged data as if all models were operating simultaneously. This alternative predicts a negative correlation between the context models, reflecting the trade-off in their activation. No evidence was found for such a trade-off, as correlation between context models were generally neutral or positive across subjects and across time (Figure 7).

A

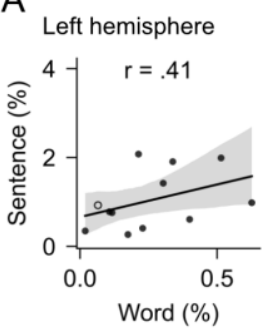

- Right-hander - Left-hander

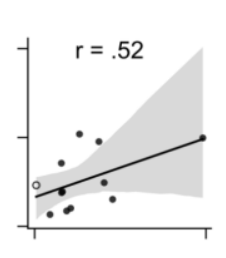

\section{ํํำ}

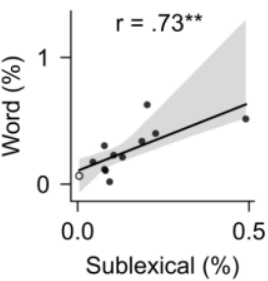

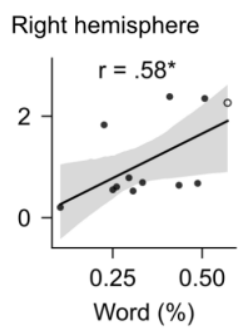
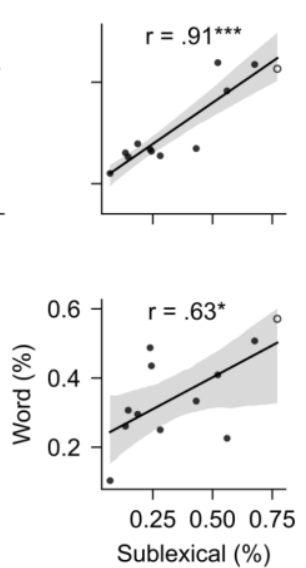

B

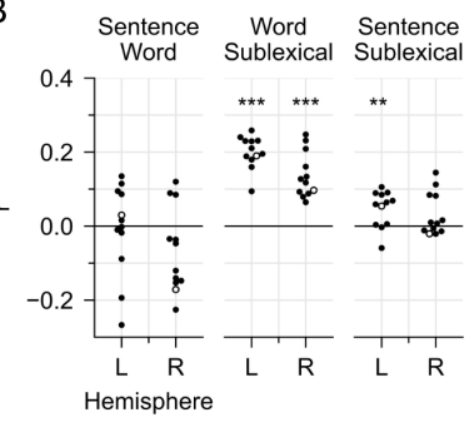

495

496

497

498

499

500

501

502

503

504

505

506

507

508

509

510

Figure 7. No evidence for a trade-off between context models.

(A) Trade-off across subjects: Testing the hypothesis that subjects differ in which context model they rely on. Each plot compares the predictive power of two context models in the $\mathrm{mid} /$ posterior STG ROI, each dot representing \% explained for one subject. The line represents a linear regression with 95\% bootstrap confidence interval (Waskom, 2021). None of the pairwise comparisons exhibits a negative correlation that would be evidence for a trade-off between reliance on different context models. Data from Figure 4-Source Data 2.txt.

(B) Trade-off over time: Testing the hypothesis that subjects alternate over time in which context model they rely on. Each dot represents the partial correlation over time between the predictive power of two context models for one subject, controlling for predictive power of the full model. Correlations are shown separately for the left and the right hemisphere $(\mathrm{L} / \mathrm{R})$. Stars correspond to a one-sample $t$-tests of the null-hypothesis that the average $r$ across subjects is 0 , i.e., that the two context models are unrelated over time. None of the context models exhibited a significant negative correlation that would be evidence for a trade-off over time. Data in Figure 7-Source Data 1.txt. 
512 The present MEG data provide clear evidence for the existence of a neural representation of 513 speech that is unified across representational hierarchies. This representation incrementally in514 tegrates phonetic input with information from the multi-word context within about 100 ms. 515 However, in addition to this globally unified representation, brain responses also show evidence 516 of separate neural representations that use more local contexts to process the same input.

517 Parallel representations of speech using different levels of context

518 The evidence for a unified global model suggests that there is a functional brain system that pro519 cesses incoming phonemes while building a representation that incorporates constraints from 520 the multi-word context. A possible architecture for such a system is the unified global architec521 ture shown in Figure 1-C, in which a probabilistic representation of the lexical cohort mediates 522 between sentence and phoneme level representations: the sentence context modifies the prior 523 expectation for each word, which is in turn used to make low-level predictions about the phonet524 ic input. While there are different possible implementations for such a system, the key feature is 525 that the global sentence context is used to make predictions for and interpret low-level phonet526 ic, possibly even acoustic (Sohoglu and Davis, 2020) input.

527 A second key result from this study, however, is evidence that this unified model is not the only 528 representation of speech. Brain responses also exhibited evidence for two other, separate func529 tional systems that process incoming phonemes while building representations that incorporate 530 different, more constrained kinds of context: one based on a local word context, processing the 531 current word with a prior based on context-independent lexical frequencies, and another based 532 on the local phoneme sequence regardless of word boundaries. Each of these three functional 533 systems generates its own predictions for upcoming phonemes, resulting in parallel responses to 534 phoneme entropy. Each system is updated incrementally at the phoneme rate, reflected in early 535 responses to surprisal. However, each system engages an at least partially different configuration 536 of neural sources, as evidenced by the localization results.

537 Together, these results suggest that multiple predictive models process speech input in parallel. 538 An architecture consistent with these observations is sketched in Figure 8: three different neural 539 systems receive the speech input in parallel. Each representation is updated incrementally by 540 arriving phonemes. However, the three systems differ in the extent and kind of context that they 541 incorporate, each generating its own probabilistic beliefs about the current word and/or future 542 phonemes. For instance, the sublexical model uses the local phoneme history to predict upcom543 ing phonemes. The updates are incremental because the state of the model at time $k+1$ is de544 termined by the state of the model at time $k$ and the phoneme input from time $k$. The same in545 cremental update pattern applies to the sublexical, word and sentence models. 


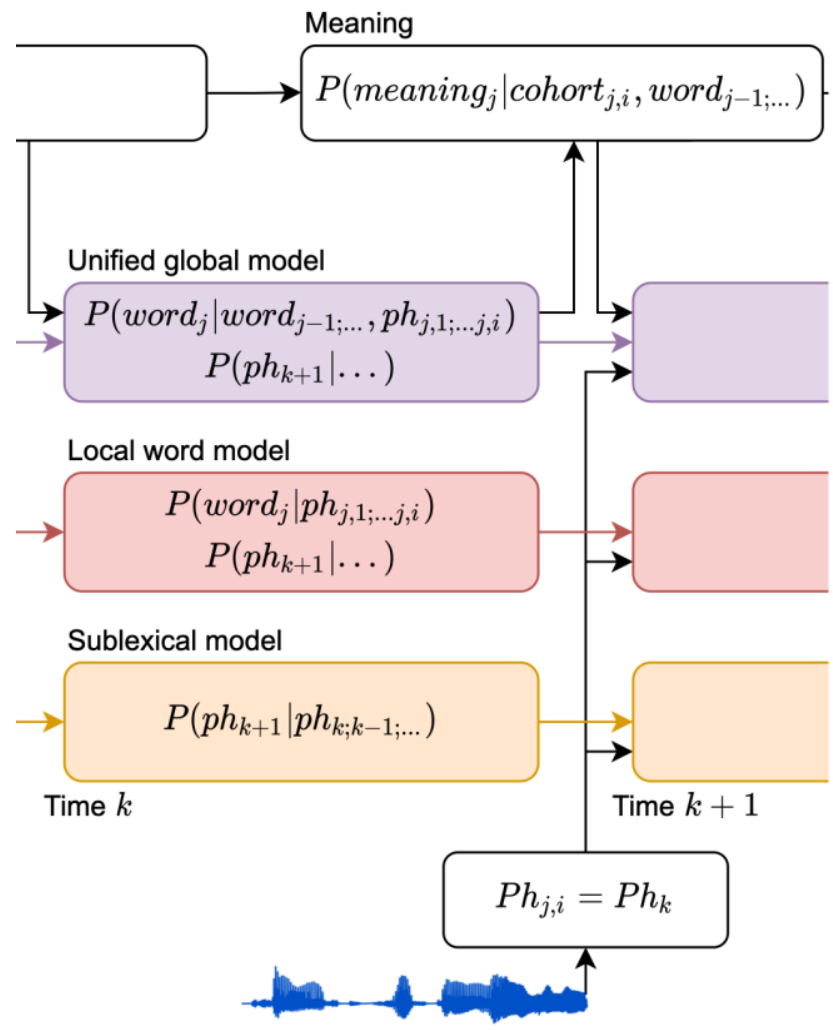

547 Figure 8. An architecture for speech perception with multiple parallel context models. A model of 548 information flow, consistent with brain signals reported here. Brain responses associated with 549 Information theoretic variables provided separate evidence for each of the probability distribu550 tions in the colored boxes. From left to right, the three different context models (sentence, lexi551 cal and sublexical) update incrementally as each phoneme arrives. The cost of these updates is 552 reflected in the brain response related to surprisal. Representations also include probabilistic 553 representations of words and upcoming phonemes, reflected in brain responses related to en554 tropy.

555 A listener whose goal is comprehending a discourse-level message might be expected to rely 556 primarily on the unified, sentence constrained context model. Consistent with this, there is some 557 evidence that this model has a privileged status. Among the linguistic models, the unified model 558 has the most explanatory power and clearly bilateral representation (Figure 4). In addition, while 559 activity in local models was short-lived, the unified model was associated with extended activa560 tion for up to $600 \mathrm{~ms}$ and recruitment of more ventral regions of the temporal lobe (Figure 5). 561 This suggests that the update in the unified model is normally more extensive than the local 562 models, and could indicate that the unified model most commonly drives semantic as well as 563 form representations, while the short-lived local models might be restricted to form-based rep564 resentations.

565 Implications for speech processing

566 A longstanding puzzle in the comprehension literature has been why activation of candidates not 567 supported by context is sometimes reported (Swinney, 1979; Zwitserlood, 1989), if top-down 
sentence context rapidly feeds down to early levels of speech perception. Parallel activation of lexical candidates based on sentence and word context models can explain these findings. Shortlived brain responses (up to $150 \mathrm{~ms}$ after phoneme onset) show evidence of parallel activation of sentence-constrained as well as sentence-independent word candidates. The co-existence of these two candidate sets can explain short-lived priming of sentence-inappropriate candidates. Whereas brain responses related to sentence-independent candidates are transient, brain responses related to sentence-appropriate candidates exhibit a secondary, sustained response (150-550 ms), explaining selective priming of sentence-appropriate candidates at longer delays.

If context-constrained candidates are immediately available, then why maintain alternative, sentence-independent candidates or even sublexical probabilistic phoneme sequences? One functional advantage might be faster recovery when sentence-based predictions turn out to be misleading. Such an effect has been described in reading, where contextually unexpected continuations are not associated with a proportional increase in processing cost (Frisson et al., 2017; Luke and Christianson, 2016).

Similarly, a representation of sublexical phoneme sequences might be functionally relevant when encountering input that is noisy or not yet part of the lexicon. Phoneme transition probabilities are generally higher within words than between words, such that low probability phoneme transitions are cues to word boundaries (Cairns et al., 1997; Harris, 1955). Statistical phoneme sequence models might thus play an important role in language acquisition by bootstrapping lexical segmentation of continuous speech (Cairns et al., 1997; Chambers et al., 2003; Saffran et al., 1996). Even in adult speech perception, they might have a similar function when encountering novel words, such as domain-specific vocabularies or personal names (Norris and McQueen, 2008). Finally, the linguistic context can be highly informative for phoneme recognition (Hitczenko et al., 2020), and different levels of context might make complementary contributions.

Our results suggest that the different context models operate in parallel, without an apparent trade-off, between subjects, or over time. However, our listening condition was also relatively uniform - listening to an audiobook in quiet background. It is conceivable that the different models play more differentiated roles in different listening conditions, for example in unpredict-

\section{Implications for word recognition}

598 Perhaps the most surprising implication of our results is that multiple probabilistic cohort representations seem to be maintained in parallel. This is most directly implied by the result that two different cohort entropy predictors both explain unique variability in the data, one based on the lexical frequency of each candidate, and another based on the contextual likelihood of each candidate. This is inconsistent with models in which each lexical candidate is assigned a single "activation" value (e.g. Morton, 1969). It might be more readily reconciled with models that distinguish between a lexical item's entry in long-term memory, and its instantiation as a token for parsing a speech signal, since such a mechanism allows for multiple tokens corresponding to the same lexical item (McClelland and Elman, 1986; Norris, 1994; Norris and McQueen, 2008). Yet, existing models generally are restricted to a single "arena" for lexical competition, whereas our results imply the possibility that the competition plays out in parallel in at least partially different 609 brain systems. 
610 A second implication is that feedback from the sentence level context can and does affect pho611 neme processing. The observed phoneme entropy effects indicate that phoneme level expecta612 tions are modulated by the sentence context. This is inconsistent with some models of word 613 recognition that assume a pure bottom-up process (e.g. Norris et al., 2000). However, at the 614 same time, the parallel architecture we propose (Figure 8) addresses a central theoretical prob615 lem associated with feedback in multi-stage architectures: Bayesian accounts of perception sug616 gest that listeners generate a prior, reflecting an estimate of future input, and compare this prior 617 to the actual input to compute a posterior probability, or interpretation of the sensory percept. 618 In multi-stage architectures that allow different priors at sequential hierarchical levels (such as 619 Figure 1-B), higher levels receive the posterior interpretation of the input from the lower levels, 620 rather than the unbiased input itself. This is suboptimal when considering a Bayesian model of 621 perception, because the prior of lower level systems is allowed to distort the bottom-up evi622 dence before it is compared to the prior generated by higher levels (Norris et al., 2016). In con623 trast, the parallel representations favored by the evidence presented here allows undistorted 624 bottom-up information to be directly compared with the context model for each definition of 625 context. The parallel model can thus accommodate empirical evidence for feedback while avoid626 ing this theoretical problem associated with sequential models.

\section{Evidence for graded linguistic predictions}

628 There is broad agreement that language processing involves prediction, but the exact nature of 629 these predictions is more controversial (DeLong et al., 2005; Huettig, 2015; Nieuwland et al., 630 2020, 2018; Pickering and Gambi, 2018). Much of the debate is about whether humans can rep631 resent distributions over many likely items, or just predict specific items. Previous research 632 showing an early influence of sentence context on speech processing has typically relied on spe633 cifically designed, highly constraining contexts which are highly predictive of a specific lexical 634 item (Holcomb and Neville, 1991; Connolly and Phillips, 1994; Van Petten et al., 1999; Rommers 635 et al., 2013). In such highly predictive contexts, listeners might indeed predict specific items, and 636 such predictions might be linked to the left-lateralized speech productions system (Federmeier, 637 2007; Pickering and Gambi, 2018). However, such a mechanism would be less useful in more 638 representative language samples, in which highly predictable words are rare (Luke and Christian639 son, 2016). In such situations of limited predictability, reading time data suggest that readers in640 stead make graded predictions, over a large number of possible continuations (Luke and Chris641 tianson, 2016; Smith and Levy, 2013). Alternatively, it has also been suggested that what looks 642 like graded predictions could actually be pre-activation of specific higher-level semantic and syn643 tactic features shared among the likely items (Altmann and Kamide, 1999; Luke and Christianson, 644 2016; Matchin et al., 2018; Pickering and Gambi, 2018; Van Berkum et al., 2005), without involv645 ing prediction of form-based representations. The present results, showing brain responses re646 flecting sentence-constrained cohort- and phoneme entropy, provide a new kind of evidence in 647 favor of graded probabilistic and form-based predictions at least down to the phoneme level.

648 Bilateral pathways to speech comprehension

649 Our results suggest that lexical/phonetic processing is largely bilateral. This is consistent with ex650 tensive clinical evidence for bilateral receptive language ability (Gazzaniga and Sperry, 1967; Ku- 
651 tas et al., 1988; Poeppel, 2001; Hickok and Poeppel, 2007), and suggestions that the right hemi652 sphere might even play a distinct role in complex, real-world language processing (Federmeier et 653 al., 2008; Jung-Beeman, 2005). In healthy participants, functional lateralization of sentence pro654 cessing has been studied in reading using visual half-field presentation (Federmeier and Kutas, 655 1999). Overwhelmingly, results from these studies suggest that lexical processing in both hemi656 spheres is dominated by sentence meaning (Coulson et al., 2005; Federmeier et al., 2005; 657 Federmeier and Kutas, 1999; Wlotko and Federmeier, 2007). This is consistent with the strong 658 bilateral representation of the unified model of speech found here. As in the visual studies, the 659 similarity of the response latencies in the two hemispheres implies that right-hemispheric effects 660 are unlikely to be due to inter-hemispheric transfer from the left hemisphere (Figure 5).

661 In addition to bilateral representation, however, our results also suggest that the two hemi662 spheres differ with respect to the context models they entertain. Visual half-field reading studies 663 have indicated a pattern of hemispheric differences, which has been interpreted as indicating 664 that the left hemisphere processes language in a maximally context-sensitive manner, whereas 665 the right hemisphere processes the sensory input in a bottom-up manner, unbiased by the lin666 guistic context (Federmeier, 2007). Our results suggest a modification of this proposal, indicating 667 that both hemispheres rely on sentence-based, graded predictions, but that the right hemi668 sphere additionally maintains stronger representations of local contexts. Finally, lateralization 669 might also depend on task characteristics such as stimulus familiarity (Brodbeck et al., 2018a), 670 and in highly constraining contexts the left hemisphere might engage the left-lateralized lan671 guage production system to make specific predictions (Federmeier, 2007; Pickering and Gambi, 672 2018).

673 Limitations of the sentence context model

674 We approximated the sentence context with a 5-gram model. This model provides an accurate 675 estimate of the sum of local constraints, based on a context of the four preceding words only. 676 However, it misses the more subtle influences of the larger context, both semantic constraints 677 and syntactic long-range dependencies, which might make the sentence level even more differ678 ent from the local context models. Furthermore, lexical N-gram models conflate the influence of 679 syntactic, semantic, and associative constraints (e.g., idioms). It is thus possible that work with 680 more sophisticated language models can reveal an even more complex relationship between 681 global and local contexts.

\section{Conclusions}

683 Prior research on the use of context during language processing has often focused on binary dis684 tinctions, such as asking whether context is or is not used to predict future input. Such questions 685 assumed a single serial or cascaded processing stream. Here we show that this assumption might 686 have been misleading, because different predictive models are maintained in parallel. Our re687 sults suggest that robust speech processing is based on probabilistic predictions using different 688 context models in parallel, and cutting across hierarchical levels of representations. 
691 Twelve native speakers of English were recruited from the University of Maryland community (6 692 female, 6 male, age mean $=21$ years, range 19-23). None reported any neurological or hearing 693 impairment. According to self-report using the Edinburgh Handedness Inventory(Oldfield, 1971), 69411 were right-handed and one left-handed. All subjects provided written informed consent in 695 accordance with the University of Maryland Institutional Review Board. Subjects either received course credit $(n=4)$ or were paid for their participation $(n=8)$. This sample size is comparable to the most directly relatable previous research which either had a similar number of subjects, $\mathrm{N}=11$ (Donhauser and Baillet, 2020) or a larger number of subjects but substantially less data per subject, $\mathrm{N}=28$ with single talker stimulus duration 8 min (Brodbeck et al., 2018a).

Stimuli

Stimuli consisted in eleven excerpts from the audiobook version of The Botany of Desire by Michael Pollan (Pollan, 2001). Each excerpt was between 210 and 332 seconds long, for a total of 46 minutes and 44 seconds. Excerpts were selected to create a coherent narrative and were presented in chronological order to maximize deep processing for meaning.

Procedure

During MEG data acquisition, participants lay in a supine position. They were allowed to keep their eyes open or closed to maximize subjective comfort and allow them to focus on the primary task of listening to the audiobook. Stimuli were delivered through foam pad earphones inserted into the ear canal at a comfortably loud listening level. After each segment, participants answered 2-3 questions relating to its content and had an opportunity to take a short break.

\section{Data acquisition and preprocessing}

712 Brain responses were recorded with a 157 axial gradiometer whole head MEG system (KIT, Kanazawa, Japan) inside a magnetically shielded room (Vacuumschmelze GmbH \& Co. KG, Hanau, Germany) at the University of Maryland, College Park. Sensors (15.5 mm diameter) are uniformly distributed inside a liquid-He dewar, spaced $\sim 25 \mathrm{~mm}$ apart, and configured as first-order axial gradiometers with $50 \mathrm{~mm}$ separation and sensitivity better than $5 \mathrm{fT} \cdot \mathrm{Hz}^{-1 / 2}$ in the white noise region (>1 KHz). Data were recorded with an online $200 \mathrm{~Hz}$ low-pass filter and a $60 \mathrm{~Hz}$ notch filter at a sampling rate of $1 \mathrm{kHz}$.

Recordings were pre-processed using mne-python (Gramfort et al., 2014). Flat channels were automatically detected and excluded. Extraneous artifacts were removed with temporal signal space separation (Taulu and Simola, 2006). Data were filtered between 1 and $40 \mathrm{~Hz}$ with a zerophase FIR filter (mne-python 0.20 default settings). Extended infomax independent component analysis (Bell and Sejnowski, 1995) was then used to remove ocular and cardiac artifacts. Responses time-locked to the speech stimuli were extracted, low pass filtered at $20 \mathrm{~Hz}$ and resampled to $100 \mathrm{~Hz}$.

728

Five marker coils attached to participants' head served to localize the head position with respect to the MEG sensors. Head position was measured at the beginning and at the end of the recording session and the two measurements were averaged. The FreeSurfer (Fischl, 2012) "fsaverage" template brain was coregistered to each participant's digitized head shape (Polhemus 3SPACE 
FASTRAK) using rotation, translation, and uniform scaling. A source space was generated using four-fold icosahedral subdivision of the white matter surface, with source dipoles oriented perpendicularly to the cortical surface. Regularized minimum $\ell 2$ norm current estimates (Dale and Sereno, 1993; Hämäläinen and IImoniemi, 1994) were computed for all data using an empty room noise covariance $(\lambda=1 / 6)$. The temporal response function analysis was restricted to brain areas of interest by excluding the occipital lobe, insula and midline structures based on the "aparc" FreeSurfer parcellation (Desikan et al., 2006). Excluded areas are shaded gray in Figure 4. A preliminary analysis (see below) was restricted to the temporal lobe (superior, middle and inferior temporal gyri, Heschl's gyrus and superior temporal sulcus).

Predictor variables

Acoustic model

741 To control for brain responses to acoustic features, all models included an 8 band gammatone

742 spectrogram and an 8 band acoustic onset spectrogram (Brodbeck et al., 2020), both covering 743 frequencies from 20 to $5000 \mathrm{~Hz}$ in equivalent rectangular bandwidth (ERB) space (Heeris, 2018) 744 and scaled with exponent 0.6 (Biesmans et al., 2017).

745 Word-and phoneme segmentation

746 A pronunciation dictionary was generated by combining the Carnegie-Mellon University pronunciation dictionary with the Montreal Forced Aligner (McAuliffe et al., 2017) dictionary and adding any additional words that occurred in the stimuli. Transcripts were then aligned to the acoustic stimuli using the Montreal Forced Aligner (McAuliffe et al., 2017) version 1.0.1. All models included control predictors for word onsets (equal value impulse at the onset of each word) and phoneme onsets (equal value impulse at the onset of each non-word initial phoneme).

\section{Context-based predictors}

All experimental predictor variables consist of one value for each phoneme and were represented as a sequence of impulses at all phoneme onsets. The specific values were derived from three different linguistic context models.

757 The complete SUBTLEX-US corpus (Brysbaert and New, 2009) was transcribed by substituting the 758 pronunciation for each word and concatenating those pronunciations across word boundaries (i.e., no silence between words). Each line was kept separate since lines are unordered in the SUBTLEX corpus. The resulting phoneme sequences were then used to train a 5-gram model using KenLM (Heafield, 2011). This 5-gram model was then used to derive phoneme surprisal and entropy.

The surprisal of experiencing phoneme $p h_{k}$ at time point $k$ is inversely related to the likelihood of that phoneme, conditional on the context (measured in bits): $I\left(p h_{k}\right)=-\log _{2}\left(p\left(p h_{k} \mid\right.\right.$ context $\left.)\right)$. In the case of the 5-phone model this context consists of the preceding 4 phonemes, $p h_{k-4 ; \ldots k-1}$.

767 The entropy $H$ (Greek Eta) at phoneme position $p h_{k}$ reflects the uncertainty of what the next 768 phoneme, $p h_{k+1}$ will be. It is defined as the expected (average) surprisal at the next phoneme, $769 H_{p h}\left(p h_{k}\right)=-\sum_{p h}^{\text {phonemes }} p\left(p h_{k+1}=p h \mid\right.$ context $) \log _{2}\left(p\left(p h_{k+1}=p h \mid\right.\right.$ context $\left.)\right)$. Based on 770 the 5 -phone model, the context here is $p h_{k-3 ; . . . k}$. 
772 The lexical context model takes into account information from all phonemes that are in the same

773 word as, and precede the current phoneme (Brodbeck et al., 2018a) and is based on the cohort model of word perception (Marslen-Wilson, 1987). At word onset, the prior for each word is proportional to its frequency in the Corpus of Contemporary American English (COCA; Davies, 2015). With each subsequent phoneme, the probability for words that are inconsistent with that phoneme is set to 0 , and the remaining distribution is renormalized. Phoneme surprisal and entropy are then calculated as above, but with the context being all phonemes in the current word so far. In addition, lexical entropy is calculated at each phoneme position as the entropy in the distribution of the cohort $H_{w}\left(p h_{j, i}\right)=-\sum_{\text {word }}^{\text {lexicon }} p\left(\right.$ word $_{j}=$ word $\mid$ context $) \log _{2}\left(p\left(\right.\right.$ word $_{j}=$ word|context)) where $j$ is the index of the word, $i$ is the index of the current phoneme within word $j$, and the context consists of phonemes $p h_{j, 1 ; \ldots, j,-1 \text {. }}$

This level thus allows two different levels of representation, phonemes and words, and two corresponding entropy values, phoneme entropy and lexical entropy. Yet, we only include one version of surprisal. The reason for this is that calculating surprisal over phonemes or over words leads to identical results. This is because the $k$ th phoneme of a word, together with the cohort at phoneme $k-1$ exhaustively defines the cohort at phoneme $k$ : $p\left(\right.$ ph $\left._{k} \mid \operatorname{cohort}_{k-1}\right) \equiv p\left(\right.$ cohort $\left._{k} \mid \operatorname{cohort}_{k-1}\right)$.

\section{Sentence context model}

The sentence context model was implemented like the lexical context model, but with the addition of lexical priors based on the 5-gram word context. A 5-gram model was trained on COCA (Davies, 2015) with KenLM (Heafield, 2011). Then, at the onset of each word, the cohort was initialized with each word's prior set to its probability given the 4 preceding words in the 5-gram model.

\section{Deconvolution}

797

Deconvolution and statistical analysis were performed with Eelbrain (Brodbeck et al., 2021) and additional scripts available at https://github.com/christianbrodbeck/TRF-Tools.

Multivariate temporal response functions (mTRFs) were computed independently for each subject and each virtual current source (Brodbeck et al., 2018b; Lalor et al., 2009). The neural response at time $t, y_{t}$ was predicted jointly from $N$ predictor time series $x_{i, t}$ convolved with a corre801 sponding $\operatorname{mTRF} h_{i, \tau}$ of length $T$ :

$$
\hat{y}_{t}=\sum_{i}^{N} \sum_{\tau}^{T} h_{n, \tau} \cdot x_{i, t-\tau}
$$

802
mTRFs were generated from a basis of 50 ms wide Hamming windows centered at $T=$ $[-100, \ldots, 1000) \mathrm{ms}$. For estimating mTRFs, all responses and predictors were standardized by centering and dividing by the mean absolute value.

For estimation using 4-fold cross-validation, each subject's data were concatenated along the time axis and split into 4 contiguous segments of equal length. The mTRFs for predicting the responses in each segment (David et al., 2007) to minimize the $\ell 1$ error. The mTRF was iteratively modified based on the maximum error reduction in the training set (the steepest coordinate de- 
scent) and validated based on the error in the validation set. Whenever a training step caused an increase of error in the validation set, the TRF for the predictor responsible for the increase was frozen, and training continued until the whole mTRF was frozen. The 3 mTRFs from the 3 training runs were then averaged to predict responses in the left-out testing segment.

\section{Model comparisons}

814 Model quality was quantified through the $\ell 1$ norm of the residuals. For this purpose, the pre815 dicted responses for the 4 test segments, each based on mTRFs estimated on the other 3 segments, were concatenated again. To compare the predictive power of two models, the difference in the residuals of the two models was calculated at each virtual source dipole. This difference map was smoothed (Gaussian window, SD $=5 \mathrm{~mm}$ ) and tested for significance using a mass-univariate one-sample $t$-test with threshold-free cluster enhancement (TFCE) (Smith and Nichols, 2009) and a null distribution based on the full set of 4095 possible permutations of the 82112 difference maps. For effect size comparison we report $t_{\max }$, the largest $t$-value in the signifi822 cant $(p \leq .05)$ area.

823 The full model consisted of the following predictors: acoustic spectrogram ( 8 bands); acoustic 824 onset spectrogram ( 8 bands); word onsets; phoneme onsets; sublexical context model (phoneme 825 surprisal and phoneme entropy); lexical context model (phoneme surprisal, phoneme entropy 826 and word entropy); sentence context model (phoneme surprisal, phoneme entropy and word 827 entropy).

828 For each of the tests reported in Figure 4, mTRFs were re-estimated using a corresponding subset of the predictors in the full model. For instance, to calculate the predictive power for a given 830 level of context, the model was re-fit using all predictors except the predictors of the level under 831 investigation. Each plot thus reflects the variability that can only be explained by the level in 832 question. This is generally a conservative estimate for the predictive power because it discounts 833 any explanatory power based on variability that is shared with other predictors. In order to de834 termine the predictive power of linguistic processing in general, we also fit a model excluding all 835 eight information-theoretic predictors from the three levels combined.

836 To express model fits in a meaningful unit, the explainable variability was estimated through the 837 largest possible explanatory power of the full model (maximum across the brain of the measured 838 response minus residuals, averaged across subjects). All model fits were then expressed as \% of 839 this value. For visualization, brain maps are not masked by significance to accurately portray the 840 continuous nature of MEG source estimates.

841 Tests of lateralization

842 For spatio-temporal tests of lateralization (Figure 4-A and D) the difference map was first mor843 phed to the symmetric "fsaverage_sym" brain (Greve et al., 2013), and the data from the right 844 hemisphere was morphed to the left hemisphere. Once in this common space, a mass-univariate 845 repeated measures $t$-test with TFCE was used to compare the difference map from the left and 846 right hemisphere. 
848 To allow for univariate analyses of predictive power, an ROI was used including a region respon849 sive to all context models (white outline in Figure 4-A). This ROI was defined as the posterior 2/3 of the combined Heschl's gyrus and STG "aparc" label, separately in each hemisphere.

851 To compare relative lateralization in this ROI (Figure 4-E), the predictive power in each hemi852 sphere's ROI was rectified (values smaller than 0 were set to 0 ). The lateralization index (LI) was 853 then computed as $L I=R H /(L H+R H)$.

854 Tests of localization difference

855 A direct comparison of two localization maps can have misleading results due to cancellation between different current sources (Lütkenhöner, 2003) as well as the spatially continuous nature of MEG source estimates (Bourguignon et al., 2018). However, a test of localization difference is possible due to the additive nature of current sources (McCarthy and Wood, 1985). Specifically, for a linear inverse solver as used here, if the relative amplitude of a configuration of current sources is held constant, the topography of the resulting source localization is also unchanged. Consequently, we employed a test of localization difference that has the null hypothesis that the topography of two effect in source space is the same (McCarthy and Wood, 1985). Localization tests were generally restricted to an area encompassing the major activation seen in Figure 4, based on "aparc" labels (Desikan et al., 2006): the posterior 2/3 of the superior temporal gyrus and Heschl's gyrus combined, the superior temporal sulcus, and the middle $3 / 5$ of the middle temporal gyrus. For each map, the values in this area were extracted and $z$-scored (separately for each hemisphere). For each comparison, the two z-scored maps were subtracted, and the resulting difference map was analyzed with a one-way repeated measures ANOVA with factor source location (left hemisphere: 180 sources; right hemisphere: 176 sources). According to the null hypothesis, the two maps should be (statistically) equal, and the difference map should only contain noise. In contrast, a significant effect of source location would indicate that the difference

873 TRF analysis

874 For the analysis of the TRFs, all 12 mTRFs estimated for each subject were averaged (4 test seg875 ments * 3 training runs). TRFs were analyzed in the normalized scale that was used for model 876 estimation.

TRF time-course

878 To extract the time course of response functions, an ROI was generated including all virtual cur879 rent sources for which at least one of the three context models significantly improved the re880 sponse predictions. To allow a fair comparison between hemispheres, the ROI was made sym881 metric by morphing it to the "fsaverage_sym" brain (Greve et al., 2013) and taking the union of 882 the two hemispheres. With this ROI, the magnitude of the TRFs at each time point was then ex883 tracted as the sum of the absolute current values across source dipoles. These time courses were resampled from $100 \mathrm{~Hz}$, used for the deconvolution, to $1000 \mathrm{~Hz}$ for visualization and for more accurate peak time extraction. Peak times were determined by finding the maximum value within the early time window $(-50-150 \mathrm{~ms})$ for each subject. Time-courses were statistically compared using mass-univariate related measures $t$-tests, with a null distribution based on the maximum statistic in the 4095 permutations (no cluster enhancement). 
890 To analyze TRF localization, TRF magnitude was quantified as the summed absolute current val891 ues in three time-windows, representing early $(-50-150 \mathrm{~ms})$, mid-latency $(150-350 \mathrm{~ms})$ and 892 late (350 - $550 \mathrm{~ms}$ ) responses (see Figure 5). Maps were smoothed (Gaussian window, SD = 5 $893 \mathrm{~mm}$ ) and tested for localization differences with the same procedure as described above (Tests 894 of localization difference).

895 Analysis of trade-off between context models

896 Several analyses were performed to detect a trade-off between the use of the different context 897 models.

\section{Trade-off by subject}

899 One possible trade-off is between subjects: some subjects might rely on sentence context more 900 than local models, whereas other subjects might rely more on local models. For example, for lex901 ical processing, this hypothesis would predict that for a subject for whom the sentence context 902 model is more predictive, the lexical context model should be less and vice versa. According to 903 this hypothesis, the predictive power of the different context models should be negatively corre904 lated across subjects. To evaluate this, we computed correlations between the predictive power 905 of the different models in the in the mid/posterior STG ROI (see Figure 7-A).

906 Trade-off over time

907 A second possible trade-off is across time: subjects might change their response characteristics 908 over time to change the extent to which they rely on the lower- or higher-level context. For ex909 ample, the depth of processing of meaningful speech might fluctuate with the mental state of 910 alertness. According to this hypothesis, the predictive power of the different context models 911 should be anti-correlated over time. To evaluate this, we calculated the residuals for the differ912 ent model fits for each time point, $\operatorname{res}_{t}=a b s\left(y_{t}-\hat{y}_{t}\right)$, aggregating by taking the mean in the $913 \mathrm{mid} /$ posterior STG ROI (separately or each subject). The predictive power was calculated for 914 each model by subtracting the residuals of the model from the absolute values of the measured 915 data (i.e., the residuals of a null model without any predictor). The predictive power for each 916 level of context was then computed by subtracting the predictive power of a corresponding re917 duced model, lacking the given level of context, from the predictive power of the full model. Fi918 nally, to reduce the number of data points the predictive power was summed in 1 second bins.

919 For each subject, the trade-off between each pair of contexts was quantified as the partial corre920 lation (Vallat, 2018) between the predictive power of the two contexts, controlling for the pre921 dictive power of the full model (to control for MEG signal quality fluctuations over time). To test 922 for a significant trade-off, a one-sample $t$-test was used for the correlation between each pair of 923 contexts in each hemisphere, with the null hypothesis that the correlation over time is 0 (see 924 Figure 7-B).

925 Acknowledgements

926 CB was supported by National Science Foundation grant 1754284. 
928 Altmann G, Steedman M. 1988. Interaction with context during human sentence processing. Cognition 30:191-238. doi:10.1016/0010-0277(88)90020-0

Altmann GTM, Kamide Y. 1999. Incremental interpretation at verbs: restricting the domain of subsequent reference. Cognition 73:247-264. doi:10.1016/S0010-0277(99)00059-1

Auksztulewicz R, Myers NE, Schnupp JW, Nobre AC. 2019. Rhythmic Temporal Expectation Boosts Neural Activity by Increasing Neural Gain. J Neurosci 39:9806-9817. doi:10.1523/JNEUROSCI.0925-19.2019

Beddor PS, McGowan KB, Boland JE, Coetzee AW, Brasher A. 2013. The time course of perception of coarticulation. J Acoust Soc Am 133:2350-2366. doi:10.1121/1.4794366

Bejjanki VR, Clayards M, Knill DC, Aslin RN. 2011. Cue Integration in Categorical Tasks: Insights from Audio-Visual Speech Perception. PLOS ONE 6:e19812. doi:10.1371/journal.pone.0019812

Bell AJ, Sejnowski TJ. 1995. An Information-Maximization Approach to Blind Separation and Blind Deconvolution. Neural Comput 7:1129-1159. doi:10.1162/neco.1995.7.6.1129

Biesmans W, Das N, Francart T, Bertrand A. 2017. Auditory-Inspired Speech Envelope Extraction Methods for Improved EEG-Based Auditory Attention Detection in a Cocktail Party Scenario. IEEE Trans Neural Syst Rehabil Eng 25:402-412. doi:10.1109/TNSRE.2016.2571900

Bourguignon M, Molinaro N, Wens V. 2018. Contrasting functional imaging parametric maps: The mislocation problem and alternative solutions. Neurolmage 169:200-211. doi:10.1016/j.neuroimage.2017.12.033

Brodbeck C, Brooks TL, Das P, Reddigari S, Kulasingham JP. 2021. Eelbrain 0.35. Zenodo. doi:10.5281/zenodo.4650416

Brodbeck C, Hong LE, Simon JZ. 2018a. Rapid Transformation from Auditory to Linguistic Representations of Continuous Speech. Curr Biol 28:3976-3983.e5. doi:10.1016/j.cub.2018.10.042

Brodbeck C, Jiao A, Hong LE, Simon JZ. 2020. Neural speech restoration at the cocktail party: Auditory cortex recovers masked speech of both attended and ignored speakers. PLOS Biol 18:e3000883. doi:10.1371/journal.pbio.3000883

Brodbeck C, Presacco A, Simon JZ. 2018b. Neural source dynamics of brain responses to continuous stimuli: Speech processing from acoustics to comprehension. Neurolmage 172:162174. doi:10.1016/j.neuroimage.2018.01.042

Broderick MP, Anderson AJ, Liberto GMD, Crosse MJ, Lalor EC. 2018. Electrophysiological Correlates of Semantic Dissimilarity Reflect the Comprehension of Natural, Narrative Speech. Curr Biol 28:803-809.e3. doi:10.1016/j.cub.2018.01.080

Brysbaert M, New B. 2009. Moving beyond Kucera and Francis: a critical evaluation of current word frequency norms and the introduction of a new and improved word frequency measure for American English. Behav Res Methods 41:977-90. doi:10.3758/BRM.41.4.977

Cairns P, Shillcock R, Chater N, Levy J. 1997. Bootstrapping Word Boundaries: A Bottom-up Corpus-Based Approach to Speech Segmentation. Cognit Psychol 33:111-153. doi:10.1006/cogp.1997.0649 
Chambers CG, Tanenhaus MK, Magnuson JS. 2004. Actions and affordances in syntactic ambiguity resolution. J Exp Psychol-Learn Mem Cogn 30:687-696. doi:Doi 10.1037/02787393.30.3.687

Chambers KE, Onishi KH, Fisher C. 2003. Infants learn phonotactic regularities from brief auditory experience. Cognition 87:B69-B77. doi:10.1016/s0010-0277(02)00233-0

Christiansen MH, Chater N. 2016. The Now-or-Never bottleneck: A fundamental constraint on language. Behav Brain Sci 39:e62. doi:10.1017/S0140525X1500031X

Clark A. 2013. Whatever next? Predictive brains, situated agents, and the future of cognitive science. Behav Brain Sci 36:181-204. doi:10.1017/S0140525X12000477

Connolly JF, Phillips NA. 1994. Event-Related Potential Components Reflect Phonological and Semantic Processing of the Terminal Word of Spoken Sentences. J Cogn Neurosci 6:256266. doi:10.1162/jocn.1994.6.3.256

Coulson S, Federmeier KD, Van Petten C, Kutas M. 2005. Right Hemisphere Sensitivity to Wordand Sentence-Level Context: Evidence From Event-Related Brain Potentials. J Exp Psychol Learn Mem Cogn 31:129-147. doi:10.1037/0278-7393.31.1.129

Dale AM, Sereno MI. 1993. Improved Localizadon of Cortical Activity by Combining EEG and MEG with MRI Cortical Surface Reconstruction: A Linear Approach. J Cogn Neurosci 5:162-176. doi:10.1162/jocn.1993.5.2.162

David SV, Mesgarani N, Shamma SA. 2007. Estimating sparse spectro-temporal receptive fields with natural stimuli. Netw Comput Neural Syst 18:191-212. doi:10.1080/09548980701609235

Davies M. 2015. Corpus of Contemporary American English (COCA). doi:10.7910/DVN/AMUDUW

DeLong KA, Urbach TP, Kutas M. 2005. Probabilistic word pre-activation during language comprehension inferred from electrical brain activity. Nat Neurosci 8:1117-1121. doi:10.1038/nn1504

Desikan RS, Ségonne F, Fischl B, Quinn BT, Dickerson BC, Blacker D, Buckner RL, Dale AM, Maguire RP, Hyman BT, Albert MS, Killiany RJ. 2006. An automated labeling system for subdividing the human cerebral cortex on MRI scans into gyral based regions of interest. Neurolmage 31:968-980. doi:10.1016/j.neuroimage.2006.01.021

Diaz MT, Swaab TY. 2007. Electrophysiological differentiation of phonological and semantic integration in word and sentence contexts. Brain Res 1146:85-100. doi:10.1016/j.brainres.2006.07.034

Donhauser PW, Baillet S. 2020. Two Distinct Neural Timescales for Predictive Speech Processing. Neuron 105:385-393.e9. doi:10.1016/j.neuron.2019.10.019

Ettinger A, Linzen T, Marantz A. 2014. The role of morphology in phoneme prediction: Evidence from MEG. Brain Lang 129:14-23. doi:10.1016/j.bandl.2013.11.004

Federmeier KD. 2007. Thinking ahead: The role and roots of prediction in language comprehension. Psychophysiology 44:491-505. doi:10.1111/j.1469-8986.2007.00531.x

Federmeier KD, Kutas M. 1999. Right words and left words: electrophysiological evidence for hemispheric differences in meaning processing. Cogn Brain Res 8:373-392. doi:10.1016/S0926-6410(99)00036-1

Federmeier KD, Mai H, Kutas M. 2005. Both sides get the point: Hemispheric sensitivities to sentential constraint. Mem Cognit 33:871-886. doi:10.3758/BF03193082 
1012

1013

1014

1015

1016

1017

1018

1019

1020

1021

1022

1023

1024

1025

1026

1027

1028

1029

1030

1031

1032

1033

1034

1035

1036

1037

1038

1039

1040

1041

1042

1043

1044

1045

1046

1047

1048

1049

1050

1051

1052

1053

1054

Federmeier KD, Wlotko EW, Meyer AM. 2008. What's 'Right' in Language Comprehension: EventRelated Potentials Reveal Right Hemisphere Language Capabilities. Lang Linguist Compass 2:1-17. doi:https://doi.org/10.1111/j.1749-818X.2007.00042.x

Feldman NH, Griffiths TL, Morgan JL. 2009. The influence of categories on perception: Explaining the perceptual magnet effect as optimal statistical inference. Psychol Rev 116:752-782. doi:10.1037/a0017196

Ferreira F, Chantavarin S. 2018. Integration and Prediction in Language Processing: A Synthesis of Old and New. Curr Dir Psychol Sci 27:443-448. doi:10.1177/0963721418794491

Fischl B. 2012. FreeSurfer. Neurolmage, 20 YEARS OF fMRI20 YEARS OF fMRI 62:774-781. doi:10.1016/j.neuroimage.2012.01.021

Fodor JA. 1985. Précis of The Modularity of Mind. Behav Brain Sci 8:1-5. doi:10.1017/S0140525X0001921X

Forseth KJ, Hickok G, Rollo PS, Tandon N. 2020. Language prediction mechanisms in human auditory cortex. Nat Commun 11:5240. doi:10.1038/s41467-020-19010-6

Frisson S, Harvey DR, Staub A. 2017. No prediction error cost in reading: Evidence from eye movements. J Mem Lang 95:200-214. doi:10.1016/j.jml.2017.04.007

Friston KJ. 2010. The free-energy principle: a unified brain theory? Nat Rev Neurosci 11:127-138. doi:10.1038/nrn2787

Futrell R, Gibson E, Levy RP. 2020. Lossy-Context Surprisal: An Information-Theoretic Model of Memory Effects in Sentence Processing. Cogn Sci 44. doi:10.1111/cogs.12814

Gagnepain P, Henson RN, Davis MH. 2012. Temporal Predictive Codes for Spoken Words in Auditory Cortex. Curr Biol 22:615-621. doi:10.1016/j.cub.2012.02.015

Ganong WF. 1980. Phonetic categorization in auditory word perception. J Exp Psychol Hum Percept Perform 6:110-125. doi:10.1037/0096-1523.6.1.110

Gaston P, Lau E, Phillips C. 2020. How does(n't) syntactic context guide auditory word recognition? doi:10.31234/osf.io/sbxpn

Gaston P, Marantz A. 2017. The time course of contextual cohort effects in auditory processing of category-ambiguous words: MEG evidence for a single "clash" as noun or verb. Lang Cogn Neurosci 33:402-423. doi:10.1080/23273798.2017.1395466

Gazzaniga MS, Sperry RW. 1967. Language after section of the cerebral commissures. Brain J Neurol 90:131-148. doi:10.1093/brain/90.1.131

Gillis M, Vanthornhout J, Simon JZ, Francart T, Brodbeck C. 2021. Neural markers of speech comprehension: measuring EEG tracking of linguistic speech representations, controlling the speech acoustics. J Neurosci. doi:10.1523/JNEUROSCI.0812-21.2021

Gramfort A, Luessi M, Larson E, Engemann DA, Strohmeier D, Brodbeck C, Parkkonen L, Hämäläinen MS. 2014. MNE software for processing MEG and EEG data. Neurolmage 86:446460. doi:10.1016/j.neuroimage.2013.10.027

Greve DN, Van der Haegen L, Cai Q, Stufflebeam S, Sabuncu MR, Fischl B, Brysbaert M. 2013. A Surface-based Analysis of Language Lateralization and Cortical Asymmetry. J Cogn Neurosci 25:1477-1492. doi:10.1162/jocn_a_00405

Gwilliams L, Marantz A. 2015. Non-linear processing of a linear speech stream: The influence of morphological structure on the recognition of spoken Arabic words. Brain Lang 147:1-13. doi:10.1016/j.bandl.2015.04.006 
1055

1056

1057

1058

1059

1060

1061

1062

1063

1064

1065

1066

1067

1068

1069

1070

1071

1072

1073

1074

1075

1076

1077

1078

1079

1080

1081

1082

1083

1084

1085

1086

1087

1088

1089

1090

1091

1092

1093

1094

1095

1096

1097

Hale J. 2003. The Information Conveyed by Words in Sentences. J Psycholinguist Res 32:101-123. doi:10.1023/A:1022492123056

Hale JT. 2016. Information-theoretical Complexity Metrics. Lang Linguist Compass 10:397-412. doi:10.1111/Inc3.12196

Halle M, Stevens K. 1962. Speech recognition: A model and a program for research. IRE Trans Inf Theory 8:155-159. doi:10.1109/TIT.1962.1057686

Hämäläinen MS, Ilmoniemi RJ. 1994. Interpreting magnetic fields of the brain: minimum norm estimates. Med Biol Eng Comput 32:35-42. doi:10.1007/BF02512476

Harris ZS. 1955. From Phoneme to Morpheme. Language 31:190. doi:10.2307/411036

Heafield K. 2011. KenLM: Faster and Smaller Language Model QueriesProceedings of the 6th Workshop on Statistical Machine Translation. Edinburgh, Scotland, UK. pp. 187-197.

Heeris J. 2018. Gammatone Filterbank Toolkit.

Heller D, Parisien C, Stevenson S. 2016. Perspective-taking behavior as the probabilistic weighing of multiple domains. Cognition 149:104-120. doi:10.1016/j.cognition.2015.12.008

Hickok G, Poeppel D. 2007. The cortical organization of speech processing. Nat Rev Neurosci 8:393-402. doi:10.1038/nrn2113

Hitczenko K, Mazuka R, Elsner M, Feldman NH. 2020. When context is and isn't helpful: A corpus study of naturalistic speech. Psychon Bull Rev 27:640-676. doi:10.3758/s13423-01901687-6

Holcomb PJ, Neville HJ. 1991. Natural speech processing: An analysis using event-related brain potentials. Psychobiology 19:286-300. doi:10.3758/BF03332082

Huettig F. 2015. Four central questions about prediction in language processing. Brain Res 1626:118-135. doi:10.1016/j.brainres.2015.02.014

Jaramillo S, Zador AM. 2011. The auditory cortex mediates the perceptual effects of acoustic temporal expectation. Nat Neurosci 14:246-251. doi:10.1038/nn.2688

Jung-Beeman M. 2005. Bilateral brain processes for comprehending natural language. Trends Cogn Sci 9:512-518. doi:10.1016/j.tics.2005.09.009

Jurafsky D. 1996. A Probabilistic Model of Lexical and Syntactic Access and Disambiguation. Cogn Sci 20:137-194. doi:10.1207/s15516709cog2002_1

Kutas M, Hillyard SA, Gazzaniga MS. 1988. Processing of Semantic Anomaly by Right and Left Hemispheres of Commissurotomy Patients: Evidence from Event-Related Brain Potentials. Brain 111:553-576. doi:10.1093/brain/111.3.553

Lalor EC, Power AJ, Reilly RB, Foxe JJ. 2009. Resolving Precise Temporal Processing Properties of the Auditory System Using Continuous Stimuli. J Neurophysiol 102:349-359. doi:10.1152/jn.90896.2008

Leonard MK, Baud MO, Sjerps MJ, Chang EF. 2016. Perceptual restoration of masked speech in human cortex. Nat Commun 7:13619. doi:10.1038/ncomms13619

Levy R. 2008. Expectation-based syntactic comprehension. Cognition 106:1126-1177. doi:10.1016/j.cognition.2007.05.006

Loftus GR, Masson MEJ. 1994. Using confidence intervals in within-subject designs. Psychon Bull Rev 1:476-490. doi:10.3758/BF03210951

Luke SG, Christianson K. 2016. Limits on lexical prediction during reading. Cognit Psychol 88:2260. doi:10.1016/j.cogpsych.2016.06.002 
1098

1099

1100

1101

1102

1103

1104

1105

1106

1107

1108

1109

1110

1111

1112

1113

1114

1115

1116

1117

1118

1119

1120

1121

1122

1123

1124

1125

1126

1127

1128

1129

1130

1131

1132

1133

1134

1135

1136

1137

1138

1139

1140

1141

Luthra S, Peraza-Santiago G, Beeson K, Saltzman D, Crinnion AM, Magnuson JS. 2021. Robust Lexically Mediated Compensation for Coarticulation: Christmash Time Is Here Again. Cogn Sci 45. doi:10.1111/cogs.12962

Lütkenhöner B. 2003. Magnetoencephalography and its Achilles' heel. J Physiol-Paris 97:641658. doi:10.1016/j.jphysparis.2004.01.020

Magnuson JS, Mirman D, Luthra S, Strauss T, Harris HD. 2018. Interaction in Spoken Word Recognition Models: Feedback Helps. Front Psychol 9:369. doi:10.3389/fpsyg.2018.00369

Marslen-Wilson W, Tyler LK. 1975. Processing structure of sentence perception. Nature 257:784-786. doi:10.1038/257784a0

Marslen-Wilson WD. 1987. Functional parallelism in spoken word-recognition. Cognition, Special Issue Spoken Word Recognition 25:71-102. doi:10.1016/0010-0277(87)90005-9

Matchin W, Brodbeck C, Hammerly C, Lau E. 2018. The temporal dynamics of structure and content in sentence comprehension: Evidence from fMRI-constrained MEG. Hum Brain Mapp 40:663-678. doi:10.1002/hbm.24403

McAuliffe M, Socolof M, Mihuc S, Wagner M, Sonderegger M. 2017. Montreal Forced Aligner: Trainable Text-Speech Alignment Using Kaldilnterspeech 2017. Presented at the Interspeech 2017. ISCA. pp. 498-502. doi:10.21437/Interspeech.2017-1386

McCarthy G, Wood CC. 1985. Scalp Distributions of Event-Related Potentials - an Ambiguity Associated with Analysis of Variance Models. Electroencephalogr Clin Neurophysiol 61:S226-S227. doi:10.1016/0013-4694(85)90858-2

McClelland JL, Elman JL. 1986. The TRACE model of speech perception. Cognit Psychol 18:1-86. doi:10.1016/0010-0285(86)90015-0

McClelland JL, Rumelhart DE. 1981. An interactive activation model of context effects in letter perception: I. An account of basic findings. Psychol Rev 88:375-407. doi:10.1037/0033295X.88.5.375

Mesgarani N, Cheung C, Johnson K, Chang EF. 2014. Phonetic feature encoding in human superior temporal gyrus. Science 343:1006-1010. doi:10.1126/science.1245994

Morton J. 1969. Interaction of information in word recognition. Psychol Rev 76:165-178. doi:10.1037/h0027366

Nieuwland MS, Barr DJ, Bartolozzi F, Busch-Moreno S, Darley E, Donaldson DI, Ferguson HJ, Fu X, Heyselaar E, Huettig F, Matthew Husband E, Ito A, Kazanina N, Kogan V, Kohút Z, Kulakova $E$, Mézière D, Politzer-Ahles S, Rousselet G, Rueschemeyer S-A, Segaert K, Tuomainen J, Von Grebmer Zu Wolfsthurn S. 2020. Dissociable effects of prediction and integration during language comprehension: evidence from a large-scale study using brain potentials. Philos Trans R Soc B Biol Sci 375:20180522. doi:10.1098/rstb.2018.0522

Nieuwland MS, Politzer-Ahles S, Heyselaar E, Segaert K, Darley E, Kazanina N, Von Grebmer Zu Wolfsthurn S, Bartolozzi F, Kogan V, Ito A, Mézière D, Barr DJ, Rousselet GA, Ferguson HJ, Busch-Moreno S, Fu X, Tuomainen J, Kulakova E, Husband EM, Donaldson DI, Kohút Z, Rueschemeyer S-A, Huettig F. 2018. Large-scale replication study reveals a limit on probabilistic prediction in language comprehension. eLife 7:e33468. doi:10.7554/eLife.33468

Nieuwland MS, Van Berkum JJA. 2006. When peanuts fall in love: N400 evidence for the power of discourse. J Cogn Neurosci 18:1098-111. doi:10.1162/jocn.2006.18.7.1098

Norris D. 1994. Shortlist: a connectionist model of continuous speech recognition. Cognition 52:189-234. doi:10.1016/0010-0277(94)90043-4 
Norris D, McQueen JM. 2008. Shortlist B: A Bayesian model of continuous speech recognition. Psychol Rev 115:357-395. doi:10.1037/0033-295X.115.2.357

Norris D, McQueen JM, Cutler A. 2016. Prediction, Bayesian inference and feedback in speech recognition. Lang Cogn Neurosci 31:4-18. doi:10.1080/23273798.2015.1081703

Norris D, McQueen JM, Cutler A. 2000. Merging information in speech recognition: Feedback is never necessary. Behav Brain Sci 23:299-325. doi:10.1017/S0140525X00003241

Oldfield RC. 1971. The assessment and analysis of handedness: The Edinburgh inventory. Neuropsychologia 9:97-113. doi:10.1016/0028-3932(71)90067-4

Pickering MJ, Gambi C. 2018. Predicting while comprehending language: A theory and review. Psychol Bull 144:1002-1044. doi:10.1037/bul0000158

Poeppel D. 2001. Pure word deafness and the bilateral processing of the speech code. Cogn Sci 25:679-693. doi:10.1207/s15516709cog2505_3

Pollan M. 2001. The Botany of Desire: A Plant's-Eye View of the World. Random House Publishing Group.

Rommers J, Meyer AS, Praamstra P, Huettig F. 2013. The contents of predictions in sentence comprehension: Activation of the shape of objects before they are referred to. Neuropsychologia 51:437-447. doi:10.1016/j.neuropsychologia.2012.12.002

Saffran JR, Aslin RN, Newport EL. 1996. Statistical Learning by 8-Month-Old Infants. Science 274:1926-1928. doi:10.1126/science.274.5294.1926

Salverda AP, Dahan D, McQueen JM. 2003. The role of prosodic boundaries in the resolution of lexical embedding in speech comprehension. Cognition 90:51-89. doi:10.1016/S00100277(03)00139-2

Schmitt L-M, Erb J, Tune S, Rysop A, Hartwigsen G, Obleser J. 2020. Predicting speech from a cortical hierarchy of event-based timescales. bioRxiv 2020.12.19.423616. doi:10.1101/2020.12.19.423616

Singer Y, Teramoto Y, Willmore BD, Schnupp JW, King AJ, Harper NS. 2018. Sensory cortex is optimized for prediction of future input. eLife 7. doi:10.7554/eLife.31557

Smith NJ, Levy R. 2013. The effect of word predictability on reading time is logarithmic. Cognition 128:302-319. doi:10.1016/j.cognition.2013.02.013

Smith SM, Nichols TE. 2009. Threshold-free cluster enhancement: Addressing problems of smoothing, threshold dependence and localisation in cluster inference. Neurolmage 44:83-98. doi:10.1016/j.neuroimage.2008.03.061

Sohoglu E, Davis MH. 2020. Rapid computations of spectrotemporal prediction error support perception of degraded speech. eLife 9:e58077. doi:10.7554/eLife.58077

Swinney DA. 1979. Lexical access during sentence comprehension: (Re)consideration of context effects. J Verbal Learn Verbal Behav 18:645-659. doi:10.1016/S0022-5371(79)90355-4

Tabas A, von Kriegstein K. 2021. Adjudicating Between Local and Global Architectures of Predictive Processing in the Subcortical Auditory Pathway. Front Neural Circuits 15. doi:10.3389/fncir.2021.644743

Tanenhaus MK, Spivey-Knowlton MJ, Eberhard KM, Sedivy JC. 1995. Integration of visual and linguistic information in spoken language comprehension. Science 268:1632-1634. doi:10.1126/science. 7777863 
Taulu S, Simola J. 2006. Spatiotemporal signal space separation method for rejecting nearby interference in MEG measurements. Phys Med Biol 51:1759. doi:10.1088/00319155/51/7/008

Vallat R. 2018. Pingouin: statistics in Python. J Open Source Softw 3:1026. doi:10.21105/joss.01026

Van Berkum JJA, Brown CM, Zwitserlood P, Kooijman V, Hagoort P. 2005. Anticipating Upcoming Words in Discourse: Evidence From ERPs and Reading Times. J Exp Psychol Learn Mem Cogn 31:443-467. doi:10.1037/0278-7393.31.3.443

van Berkum JJA, Zwitserlood P, Hagoort P, Brown CM. 2003. When and how do listeners relate a sentence to the wider discourse? Evidence from the N400 effect. Cogn Brain Res 17:701718. doi:10.1016/S0926-6410(03)00196-4

Van Petten C, Coulson S, Rubin S, Plante E, Parks M. 1999. Time course of word identification and semantic integration in spoken language. J Exp Psychol Learn Mem Cogn 25:394-417. doi:10.1037//0278-7393.25.2.394

Vitevitch MS, Luce PA. 1999. Probabilistic Phonotactics and Neighborhood Activation in Spoken Word Recognition. J Mem Lang 40:374-408. doi:10.1006/jmla.1998.2618

Vitevitch MS, Luce PA. 1998. When Words Compete: Levels of Processing in Perception of Spoken Words. Psychol Sci 9:325-329. doi:10.1111/1467-9280.00064

Waskom M. 2021. seaborn: statistical data visualization. Zenodo. doi:10.5281/zenodo.4645478

Weissbart H, Kandylaki KD, Reichenbach T. 2020. Cortical Tracking of Surprisal during Continuous Speech Comprehension. J Cogn Neurosci 32:155-166. doi:10.1162/jocn_a_01467

Willems RM, Frank SL, Nijhof AD, Hagoort P, van den Bosch A. 2016. Prediction During Natural Language Comprehension. Cereb Cortex 26:2506-2516. doi:10.1093/cercor/bhv075

Wilson SM, Bautista A, McCarron A. 2018. Convergence of spoken and written language processing in the superior temporal sulcus. Neurolmage 171:62-74. doi:10.1016/j.neuroimage.2017.12.068

Wlotko EW, Federmeier KD. 2007. Finding the right word: Hemispheric asymmetries in the use of sentence context information. Neuropsychologia 45:3001-3014. doi:10.1016/j.neuropsychologia.2007.05.013

Zwitserlood P. 1989. The locus of the effects of sentential-semantic context in spoken-word processing. Cognition 32:25-64. doi:10.1016/0010-0277(89)90013-9 
Local context models

Sentence level

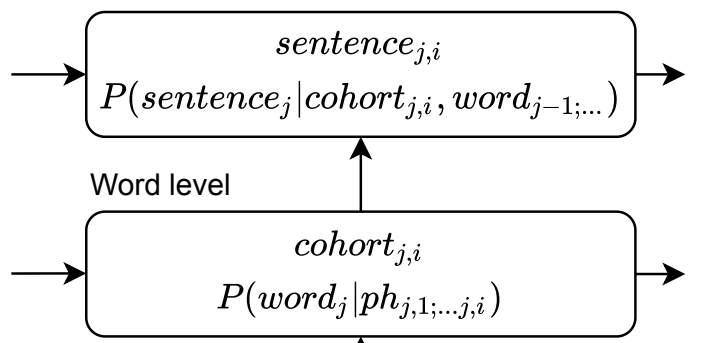

Sublexical level

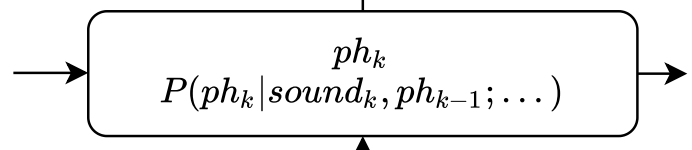

C Unified global context model

Sentence level

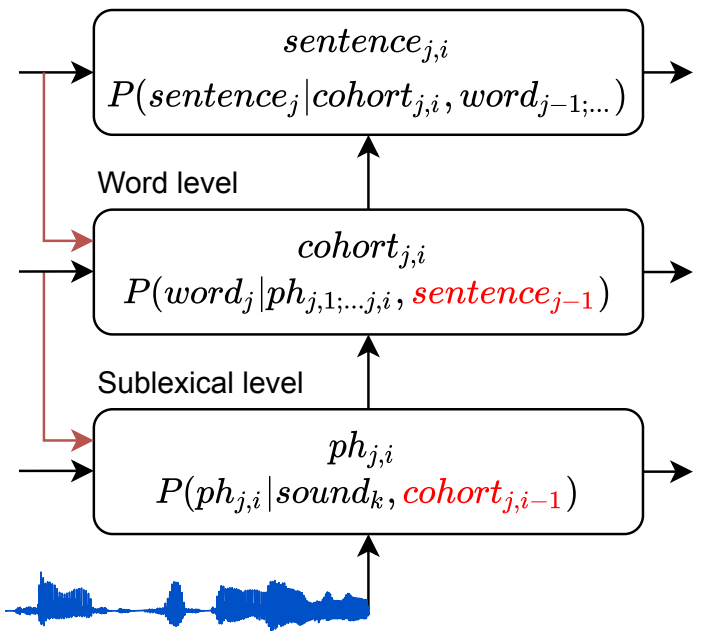




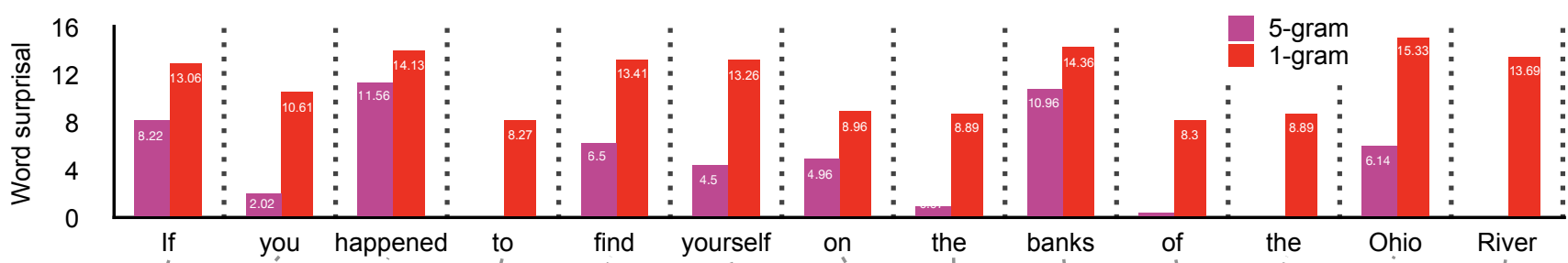

B

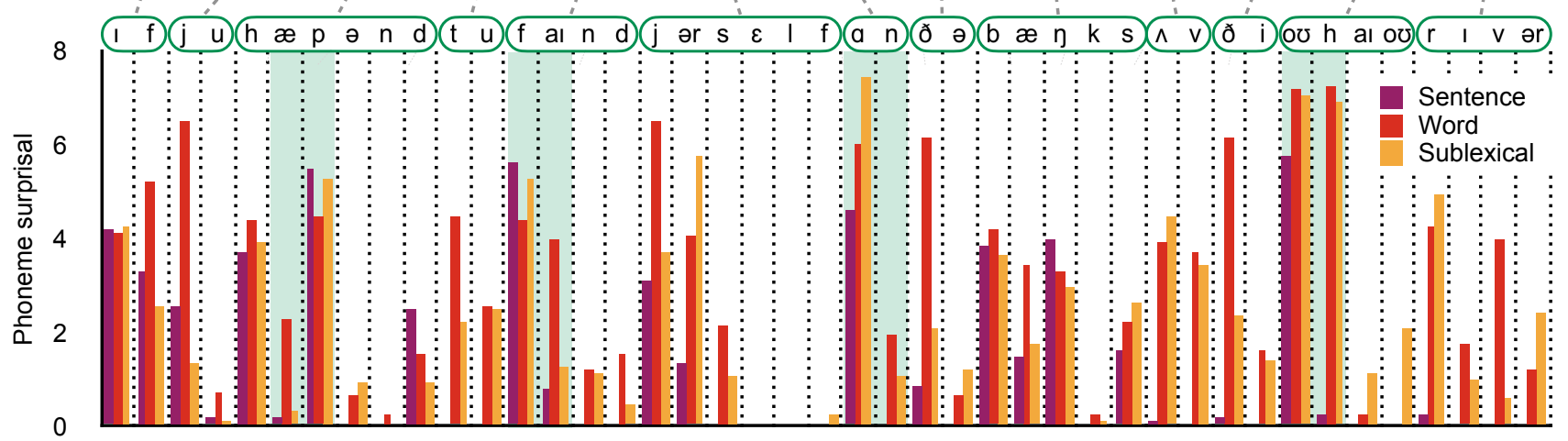

C

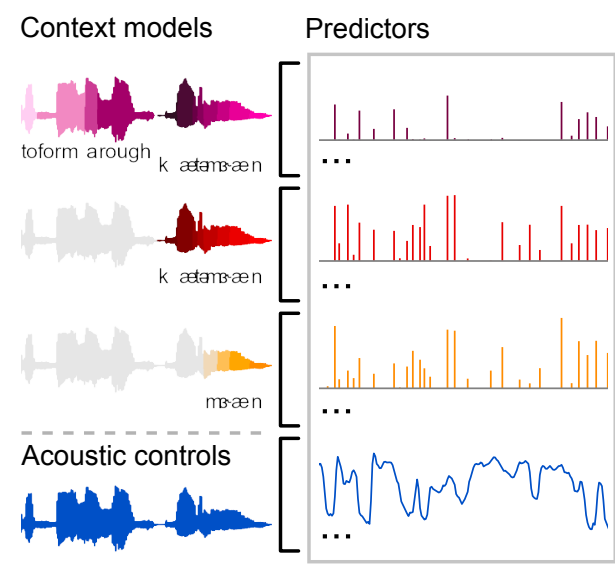

Experiment time

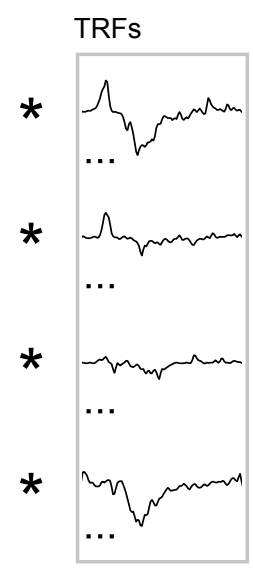

Delay

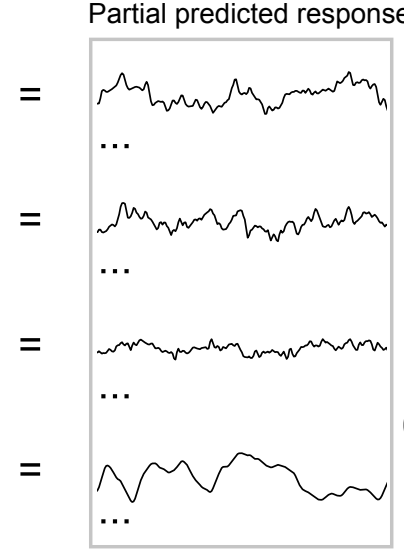

Experiment time $\longrightarrow$

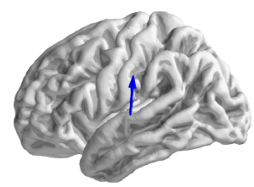

Measured response

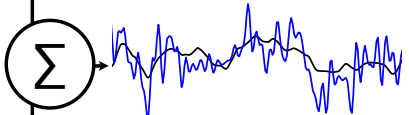

Predicted response 

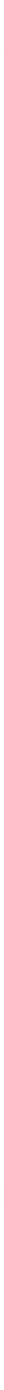
A Surprisal-TRF magnitude

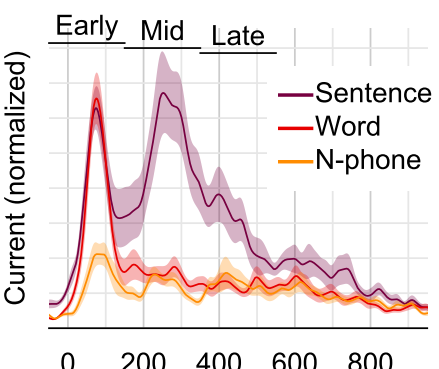

C Lexical cohort entropy

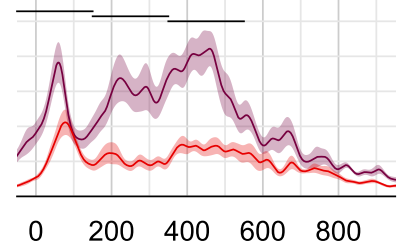

E Phoneme entropy

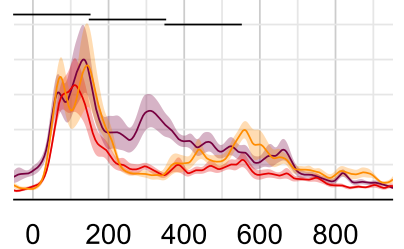

B TRF magnitude by hemisphere

—Left hemisphere

----Right hemisphere

Sentence context

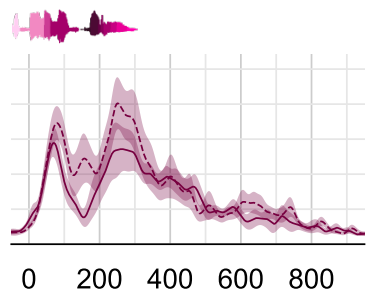

D

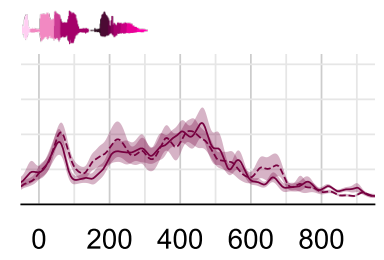

F

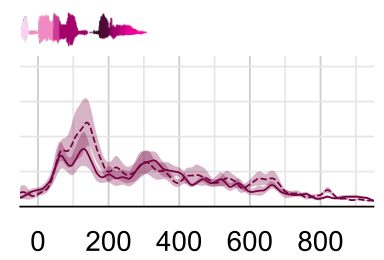

Word context
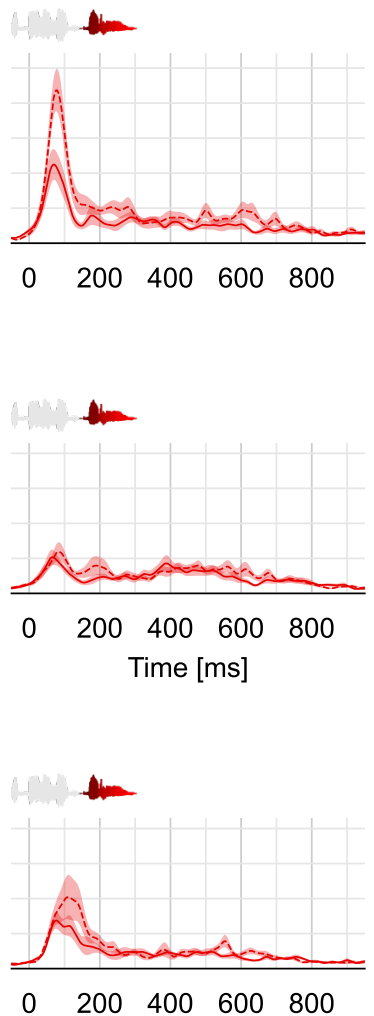

Sublexical context

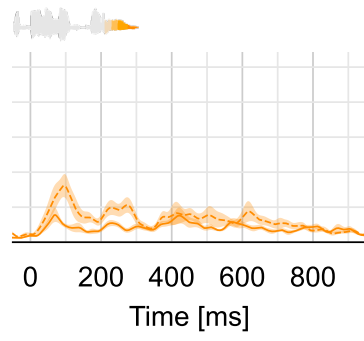

G

Sentence context

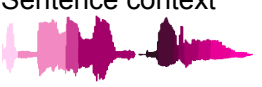

Word context

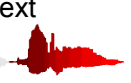

Sublexical context
Early
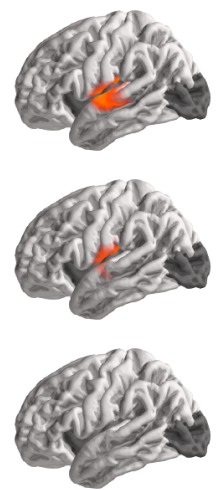
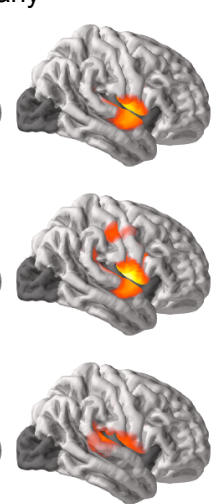

Mid

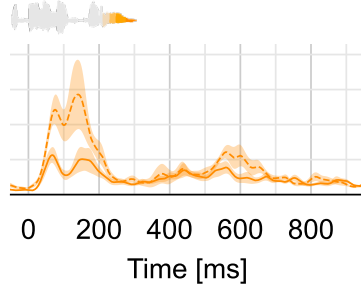

Late
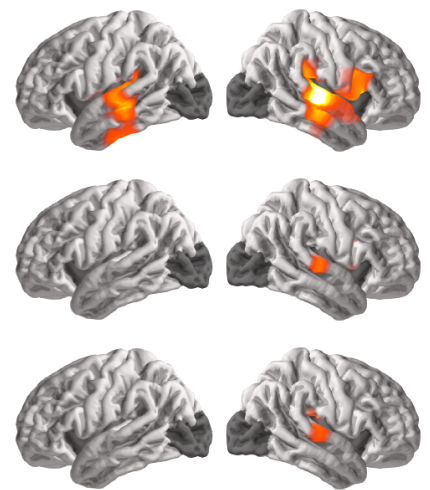

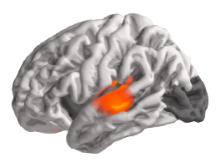

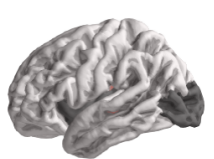
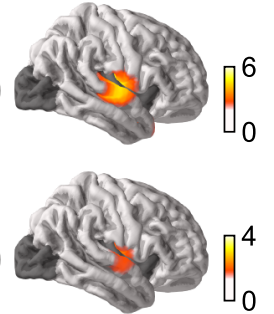

के
ำ
है
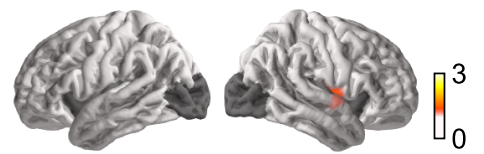
A Left hemisphere

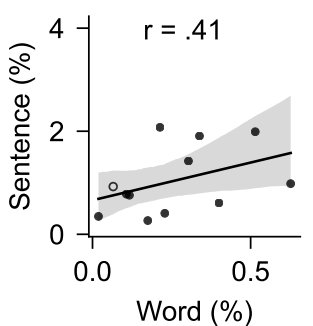

- Right-hander

- Left-hander

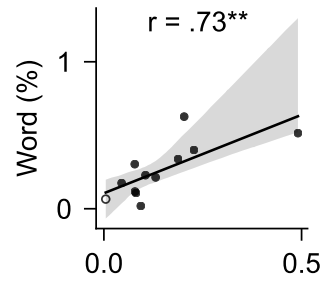

Sublexical (\%)

\section{Right hemisphere}
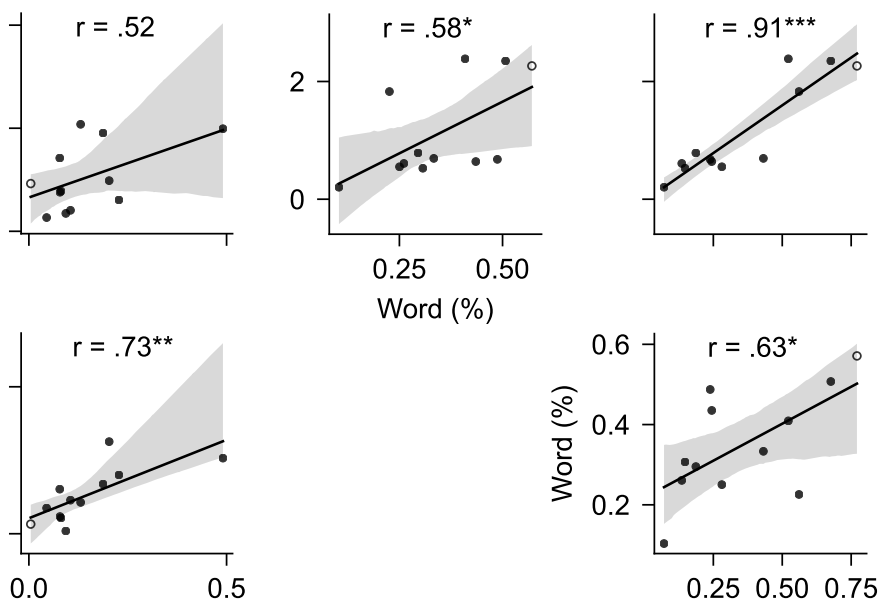

Sublexical (\%)
B

Sentence Word Sentence

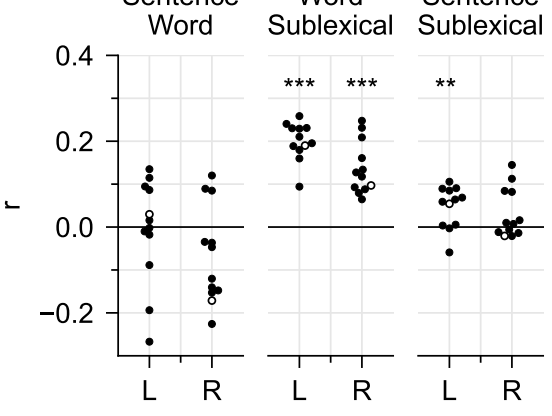

Hemisphere 
$P\left(\right.$ meaning $_{j} \mid$ cohort $_{j, i}$, word $\left._{j-1 ; \ldots}\right)$

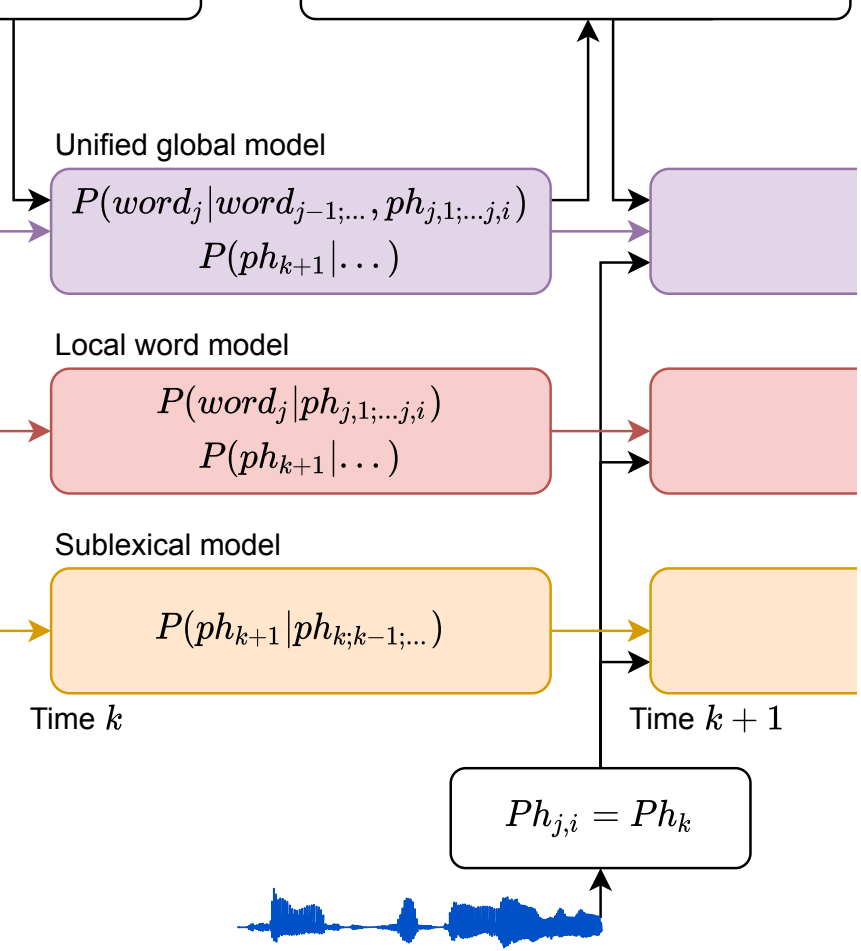

